\title{
$h p$-Version discontinuous Galerkin methods on polygonal and polyhedral meshes
}

\author{
ANDREA CANGIANI \\ Department of Mathematics, University of Leicester, University Road, \\ Leicester LE1 7RH, United Kingdom \\ Andrea.Cangiani@le.ac.uk \\ EMMANUIL H. GEORGOULIS \\ Department of Mathematics, University of Leicester, University Road, \\ Leicester LE1 7RH, United Kingdom \\ Emmanuil.Georgoulis@le.ac.uk \\ PAUL HOUSTON* \\ School of Mathematical Sciences, University of Nottingham, University Park, \\ Nottingham, NG7 2RD, United Kingdom \\ Paul.Houston@nottingham.ac.uk
}

Received (Day Month Year)

Revised (Day Month Year)

Communicated by (xxxxxxxxxx)

\begin{abstract}
An $h p$-version interior penalty discontinuous Galerkin method (DGFEM) for the numerical solution of second-order elliptic partial differential equations on general computational meshes consisting of polygonal/polyhedral elements is presented and analysed. Utilizing a bounding box concept, the method employs elemental polynomial bases of total degree $p$ ( $\mathcal{P}_{p}$-basis) defined on the physical space, without the need to map from a given reference or canonical frame. This, together with a new specific choice of the interior penalty parameter which allows for face-degeneration, ensures that optimal $a$ priori bounds may be established, for general meshes including polygonal elements with degenerating edges in two dimensions and polyhedral elements with degenerating faces and/or edges in three dimensions. Numerical experiments highlighting the performance of the proposed method are presented. Moreover, the competitiveness of the $p$-version DGFEM employing a $\mathcal{P}_{p}$-basis in comparison to the conforming $p$-version finite element method on tensor-product elements is studied numerically for a simple test problem.
\end{abstract}

Keywords: Discontinuous Galerkin; polygonal elements; polyhedral elements; $h p$-finite element methods; inverse estimates; $P$-basis.

AMS Subject Classification: 65N30, 65N50, 65N55

*Supported by the Leverhulme Trust. 


\section{Introduction}

The origins of the Discontinuous Galerkin Finite Element Method (DGFEM, for short) can be traced back almost half a century ago to the work undertaken on the weak enforcement of Dirichlet boundary conditions for second-order elliptic partial differential equations; see, for example, Refs. 15, 19, 53, 58. In particular, we highlight the works of Nitsche and Baker, see Refs. 58, 20, respectively, which form the basis of the now popular class of so-called interior penalty (IP) DGFEMs, cf. also Refs. 11, 70. Independently, DGFEMs were formulated for the numerical solution of first-order hyperbolic problems in the early 1970s by Reed \& Hill in Ref. 60; see also Ref. 52. Despite this early progress on DGFEMs, much of the subsequent research in the field of numerical analysis of partial differential equations concentrated on the development and analysis of conforming finite element methods for self-adjoint elliptic problems, stabilized continuous finite element methods for convection-diffusion equations, and finite difference and finite volume methods for hyperbolic problems. However, the past couple of decades has witnessed a resurgence of interest in discontinuous schemes. Indeed, tremendous progress has been made on both the analytical and computational aspects of DGFEMs; for a review of some of the main developments in the subject, we refer to the recent monographs in Refs. 38, 39, 46, 61. For a historical review of DGFEMs, we refer to the articles in Refs. 14, 37.

This paradigm shift has been stimulated by a number of important factors. In the context of nonlinear hyperbolic conservation laws, DGFEMs have a number of important advantages over well established finite volume methods. The concept of higher-order discretization is inherent to the DGFEM. Moreover, the stencil is minimal in the sense that each element communicates only with its direct neighbours. In particular, in contrast to the increasing stencil size needed to increase the accuracy of classical finite volume methods, the stencil of DGFEMs is the same for any order of accuracy which has important advantages for the implementation of boundary conditions and for the parallel efficiency of the method. As for finite volume methods, DGFEMs are, by construction, locally conservative. Moreover, DGFEMs can naturally treat convection-dominated diffusion problems without excessive numerical stabilization in a unified manner. Due to the simple communication at element interfaces afforded by DGFEMs, elements with so-called hanging nodes can easily be treated, a fact that simplifies local mesh refinement ( $h$-refinement); this is a major advantage of DGFEMs when compared to standard Galerkin (conforming) finite element methods (CGFEMs, for short). Indeed, this latter class of methods must exploit appropriate inter-element projections in order to ensure that the resulting finite element space satisfies the underlying continuity constraints inherent in the physical problem at hand, cf. Ref. 64, for example. Additionally, the communication at element interfaces is identical for any order of the method which simplifies the use of schemes with different polynomial orders $p$ in adjacent elements. This allows for the variation of the order of polynomials over 
the computational domain ( $p$-refinement), which in combination with $h$-refinement leads to so-called $h p$-adaptivity. Finally, in the context of domain decomposition preconditioners, DGFEMs have significant advantages over CGFEMs. Indeed, in the recent work undertaken by Antonietti and collaborators, see Refs. 4, 5, 6, 9, 10, for example, it is demonstrated that Schwarz-type preconditioners are particularly well-suited to DGFEMs, in the sense that uniform scalability of the underlying iterative method may be established without the need to overlap the subdomain partition of the computational mesh. This is a particularly attractive property, since the absence of overlapping subdomains reduces communication between processors on parallel machines. By (uniform) scalability, we mean that the number of iterations needed to compute the solution of the underlying system of equations is uniform, as the mesh is refined, provided that an appropriate coarse mesh solution is computed as part of the preconditioning strategy. Of course, for uniformity, the ratio of the granularity of the fine and coarse meshes must remain fixed under mesh refinement.

The major criticism of DGFEMs compared with their CGFEM counterparts is the increase in the number of degrees of freedom for a particular choice of the underlying computational finite element mesh and polynomial degree distribution. Moreover, it is typically observed that the discretization error computed using both schemes with the same discretization parameters (i.e., the same $h$ and $p$ ) is roughly identical, at least, for the discretization of second-order elliptic partial differential equations; this naturally leads to the conclusion that DGFEMs are computationally expensive. Such arguments are typically made on the grounds of employing identical (mapped) elemental polynomial spaces within both numerical schemes. However, the flexibility of DGFEMs means that we are no longer restricted to employing standard polynomial spaces mapped from a reference or canonical frame. Indeed, DGFEMs may be constructed in a simple manner in the physical frame, without resorting to the use of local element mappings; see, for example, the recent work by Bassi et al. in Refs. 21, 22, 23. As noted in Ref. 21, one of the key features of employing DGFEMs based on exploiting polynomial spaces defined in the physical frame is that the order of convergence of the underlying method is independent of the element shape; see Refs. 12, 13 for a detailed discussion of this issue, when element mappings are employed. Thereby, spaces of polynomials of total degree $p$, denoted by $\mathcal{P}_{p}$, may be employed, regardless of the element shape. Indeed, as we shall see in this work, when the underlying mesh consists of tensor-product elements, e.g., quadrilaterals in $2 \mathrm{D}$ and hexahedra in $3 \mathrm{D}$, the use of $\mathcal{P}_{p}$ polynomial spaces not only renders the underlying DGFEM more efficient than the standard DGFEM using tensor-product polynomials of degree $p$ in each coordinate direction $\left(\mathcal{Q}_{p}\right)$, but also more efficient than CGFEM, as the polynomial degree $p$ increases.

The exploitation of DGFEMs using polynomial spaces defined in the physical frame, without the need to employ element mappings from a reference frame, means that DGFEMs can naturally be extended to computational meshes consisting of gen- 
eral element shapes; indeed, general polygonal/polyhedral elements may easily be admitted, cf. Refs. 8, 21, 22, 23, 39, 43, 54, 72. In particular, we mention the recent work by Bassi et al. in Refs. 21, 22, 23 on the application of DGFEMs on meshes consisting of general agglomerated elements. A closely related technique proposed in Refs. 8, 43 considers the development of a general class of so-called Composite DGFEMs; this approach allows for the numerical approximation of partial differential equations posed on complicated domains which contain 'small' geometrical features, or so-called microstructures. Moreover, by exploiting these techniques, coarse meshes can easily be constructed for application within multilevel preconditioners, cf. Refs. 7, 42. This flexibility of DGFEMs has also been exploited in the context of fictitious domain methods, whereby overlapping meshes are employed; indeed, the application of DGFEMs to the discretization of second-order elliptic partial differential equations on general cut-cell meshes has been considered in Ref. 51 . We also refer to the work presented in the series of articles in Refs. 31, 32, 33, 55 which exploit ideas from Nitsche's method in Ref. 58.

In contrast, the extension of conforming finite element methods to general meshes with polygonal/polyhedral elements is far from straightforward, due to the intrinsic difficulty encountered in the design of finite element spaces with a given continuity property. Notable examples are the Composite Finite Element Method (CFE), see Refs. 44, 45, the Polygonal Finite Element Methods (PFEM), see Refs. 67, 68, and the Extended Finite Element Method (XFEM), see Ref. 40 and the references cited therein. These latter two approaches achieve conformity by enriching/modifying the standard polynomial finite element spaces, in the spirit of the Generalized Finite Element framework of Babuška and Osborn in Ref. 16. Typically, the handling of non-standard shape functions carries an increase in computational effort. The recently proposed Virtual Element Method (VEM), see Ref. 24, overcomes this difficulty achieving the extension of CGFEM to polygonal/polyhedral elements while maintaining the ease of implementation of standard FEMs; see also the very much related Mimetic Finite Difference method in its primal, see Refs. 28, 27, and mixed, see Refs. 30, 29, 28, 34, 25, 26, formulations.

The aim of this article is to consider the application of DGFEMs on computational meshes consisting of general polygonal/polyhedral elements. In particular, we propose a new IP DGFEM characterized by a careful choice of the discontinuitypenalization parameter, which permits the use of polygonal/polyhedral elements such that

- mesh element faces may have arbitrarily small measure in two dimensions;

- both mesh element faces and edges may have arbitrarily small measure in three dimensions.

The approach is based on exploiting a new inverse inequality relevant to elements with elemental interfaces whose measure is potentially much smaller than the measure of the corresponding element. On the basis of this inverse inequality, together 
with appropriate approximation results on general polygons/polyhedra, we derive a priori error bounds for the proposed IP DGFEM; for related bounds, based on employing composite DGFEMs, we refer to Ref. 8.

The rest of this article is structured as follows. In Section 2 we introduce the model problem and the set of admissible subdivisions of the computational domain. In Section 3 we formulate the IP DGFEM; Section 4 presents relevant $h p$ approximation results and the new inverse inequality. The stability and a priori analysis of the proposed method is then undertaken in Section 5. In Section 6 we briefly outline how the proposed IP DGFEM may be efficiently implemented. The practical performance of the IP DGFEM is studied in Section 7. Finally, in Section 8 we summarize the work presented in this paper and draw some conclusions.

\section{Preliminaries}

Throughout this article, we use the following standard function spaces. For a Lipschitz domain $\omega \subset \mathbb{R}^{d}, d \geq 1$, we denote by $H^{s}(\omega)$ the Sobolev space of index $s \geq 0$ of real-valued functions defined on $\omega$, endowed with the seminorm $|\cdot|_{H^{s}(\omega)}$ and norm $\|\cdot\|_{H^{s}(\omega)}$. Furthermore, we let $L^{p}(\omega), p \in[1, \infty]$, be the standard Lebesgue space on $\omega$, equipped with the norm $\|\cdot\|_{L^{p}(\omega)}$. Finally, with $|\omega|$ we denote the $d$-dimensional Hausdorff measure of $\omega$.

\subsection{Model problem}

Let $\Omega$ be a bounded open polyhedral domain in $\mathbb{R}^{d}, d=2,3$, and let $\partial \Omega$ signify the union of its $(d-1)$-dimensional open faces. We consider the following model problem: find $u$ such that

$$
-\Delta u=f \quad \text { in } \Omega,
$$

where $f \in L^{2}(\Omega)$. We divide $\partial \Omega$ into two disjoint subsets $\Gamma_{\mathrm{D}}$ and $\Gamma_{\mathrm{N}}$ whose union is $\partial \Omega$, with $\Gamma_{\mathrm{D}}$ nonempty and relatively open in $\partial \Omega$. With this notation, we supplement (2.1) with the boundary conditions

$$
\begin{aligned}
u & =g_{\mathrm{D}} \text { on } \Gamma_{\mathrm{D}}, \\
\mathbf{n} \cdot \nabla u & =g_{\mathrm{N}} \text { on } \Gamma_{\mathrm{N}},
\end{aligned}
$$

where $\mathbf{n}$ denotes the unit outward normal vector on the boundary $\partial \Omega$.

\subsection{Finite element spaces}

Let $\mathcal{T}$ be a subdivision of the computational domain $\Omega$ into disjoint open polygonal/polyhedral elements $\kappa$ such that $\bar{\Omega}=\cup_{\kappa \in \mathcal{T}} \bar{\kappa}$ and denote by $h_{\kappa}$ the diameter of $\kappa \in \mathcal{T}$; i.e., $h_{\kappa}:=\operatorname{diam}(\kappa)$. In the absence of hanging nodes/edges, we define the interfaces of the mesh $\mathcal{T}$ to be the set of $(d-1)$-dimensional facets of the elements $\kappa \in \mathcal{T}$. To facilitate the presence of hanging nodes/edges, which are permitted in $\mathcal{T}$, the interfaces of $\mathcal{T}$ are defined to be the intersection of the $(d-1)$-dimensional 
facets of neighbouring elements. In the case when $d=2$, we note that the interfaces of a given element $\kappa \in \mathcal{T}$ will always consist of $(d-1)$-dimensional simplexes, namely, line segments. In general, for $d=3$, this will not be the case; for the purposes of the forthcoming error analysis, we assume that each interface of an element $\kappa \in \mathcal{T}$ may be subdivided by a set of co-planar triangles. With this in mind we use the terminology 'face' to refer to a $(d-1)$-dimensional simplex (line segment or triangle for $d=2,3$, respectively), which forms part of the boundary (interface) of an element $\kappa \in \mathcal{T}$. For $d=2$, the face and interface of an element $\kappa \in \mathcal{T}$ necessarily coincide with each other.

With this construction, we assume that a sub-triangulation into faces of each mesh interface is given if $d=3$. We denote by $\Gamma$ the union of all open mesh interfaces if $d=2$ and the union of all open triangles belonging to the sub-triangulation of all mesh interfaces if $d=3$. In this way, $\Gamma$ is always defined as a set of $(d-1)$-simplexes. Specific assumptions on the class of admissible meshes will be given in Section 4 .

Further, we denote by $\Gamma_{\text {int }}$ the union of all open $(d-1)$-dimensional element faces $F \subset \Gamma$ that are contained in $\Omega$. With this notation, we may write $\Gamma=\Gamma_{\mathrm{D}} \cup \Gamma_{\mathrm{N}} \cup \Gamma_{\text {int }}$, where $\Gamma_{\mathrm{D}}, \Gamma_{\mathrm{N}} \subset \partial \Omega$ and $\Gamma_{\text {int }}:=\Gamma \backslash \partial \Omega$ are disjoint.

To each element $\kappa \in \mathcal{T}$, we associate a positive integer $p_{\kappa}$, henceforth referred to as the polynomial degree of the element $\kappa$, and collect the $p_{\kappa}$ in the vector $\mathbf{p}:=\left(p_{\kappa}: \kappa \in \mathcal{T}\right)$. With this notation, we define the finite element space $S_{\mathcal{T}}^{\mathbf{p}}$ with respect to $\mathcal{T}$ and $\mathbf{p}$ by

$$
S_{\mathcal{T}}^{\mathbf{p}}:=\left\{u \in L^{2}(\Omega):\left.u\right|_{\kappa} \in \mathcal{P}_{p_{\kappa}}(\kappa), \kappa \in \mathcal{T}\right\}
$$

where $\mathcal{P}_{p}(\kappa)$ denotes the space of polynomials of total degree $p$ on $\kappa$. We stress that, by construction, the local elemental polynomial spaces employed within the definition of $S_{\mathcal{T}}^{\mathbf{p}}$ are defined in the physical space, without the need to map from a given reference or canonical frame, as is typically necessary for classical finite element methods.

\section{Interior penalty discontinuous Galerkin method}

In this section we introduce the DGFEM discretization of the model problem (2.1), (2.2). For simplicity of presentation, we consider the popular (symmetric) IP DGFEM; this will allow us to focus on the key challenges posed by the exploitation of general computational meshes consisting of polygonal/polyhedral elements.

To this end, we define some trace operators that are required for the DGFEM. Let $\kappa^{+}$and $\kappa^{-}$be two adjacent elements of $\mathcal{T}$ and let $\mathbf{x}$ be an arbitrary point on the interior face $F \subset \Gamma_{\text {int }}$ given by $F=\partial \kappa^{+} \cap \partial \kappa^{-}$. We write $\mathbf{n}_{\kappa}^{+}$and $\mathbf{n}_{\kappa}^{-}$to denote the outward unit normal vectors on $F$, relative to $\partial \kappa^{+}$and $\partial \kappa^{-}$, respectively. Furthermore, let $v$ and $\mathbf{q}$ be scalar- and vector-valued functions, respectively, that are smooth inside each element $\kappa^{ \pm}$. By $\left(v^{ \pm}, \mathbf{q}^{ \pm}\right)$, we denote the traces of $(v, \mathbf{q})$ on $F$ taken from within the interior of $\kappa^{ \pm}$, respectively. Then, the averages of $v$ and $\mathbf{q}$ 
at $\mathbf{x} \in F$ are given by

$$
\left\{\{v\}=\frac{1}{2}\left(v^{+}+v^{-}\right), \quad\{\mathbf{q}\}=\frac{1}{2}\left(\mathbf{q}^{+}+\mathbf{q}^{-}\right),\right.
$$

respectively. Similarly, the jumps of $v$ and $\mathbf{q}$ at $\mathbf{x} \in F$ are given by

$$
\llbracket v \rrbracket=v^{+} \mathbf{n}_{\kappa^{+}}+v^{-} \mathbf{n}_{\kappa^{-}}, \quad \llbracket \mathbf{q} \rrbracket=\mathbf{q}^{+} \cdot \mathbf{n}_{\kappa^{+}}+\mathbf{q}^{-} \cdot \mathbf{n}_{\kappa^{-}},
$$

respectively. On a boundary face $F \subset \Gamma_{\mathrm{D}}$, we set

$$
\left\{\{v\}=v^{+}, \quad\{\mathbf{q}\}=\mathbf{q}^{+}, \quad \llbracket v \rrbracket=v^{+} \mathbf{n},\right.
$$

with $\mathbf{n}$ denoting the unit outward normal vector on the boundary $\partial \Omega$.

The standard symmetric IP DGFEM is given by: find $u_{h} \in S_{\mathcal{T}}^{\mathbf{p}}$ such that

$$
B\left(u_{h}, v_{h}\right)=\ell\left(v_{h}\right)
$$

for all $v_{h} \in S_{\mathcal{T}}^{\mathbf{p}}$. Here, the bilinear form $B: S_{\mathcal{T}}^{\mathbf{p}} \times S_{\mathcal{T}}^{\mathbf{p}} \rightarrow \mathbb{R}$ is given by

$B(w, v):=\sum_{\kappa \in \mathcal{T}} \int_{\kappa} \nabla w \cdot \nabla v \mathrm{~d} \mathbf{x}-\int_{\Gamma \backslash \Gamma_{\mathrm{N}}}\left(\left\{\left\{\nabla_{h} w\right\} \cdot \llbracket v \rrbracket+\left\{\left\{\nabla_{h} v\right\} \cdot \llbracket w \rrbracket-\sigma \llbracket w \rrbracket \cdot \llbracket v \rrbracket\right) \mathrm{d} s\right.\right.$, and the linear functional $\ell: S_{\mathcal{T}}^{\mathbf{p}} \rightarrow \mathbb{R}$ is defined by

$$
\ell(v)=\int_{\Omega} f v \mathrm{~d} \mathbf{x}-\int_{\Gamma_{\mathrm{D}}} g_{\mathrm{D}}\left(\nabla_{h} v \cdot \mathbf{n}-\sigma v\right) \mathrm{d} s+\int_{\Gamma_{\mathrm{N}}} g_{\mathrm{N}} v \mathrm{~d} s .
$$

Furthermore, $\nabla_{h}$ denotes the elementwise gradient operator. The non-negative function $\sigma \in L^{\infty}\left(\Gamma \backslash \Gamma_{\mathrm{N}}\right)$ is referred to as the discontinuity-penalization parameter; the precise definition of $\sigma$ will be presented in the forthcoming analysis; cf. Lemma 5.1 below. Historically IP methods were the first to appear in the literature, see Refs. 20, 11, based on exploiting the ideas developed by Nitsche in Ref. 58 in the context of the weak enforcement of inhomogeneous Dirichlet boundary conditions.

\section{Approximation and inverse estimates}

Before embarking on the error analysis of the $h p$-version IP DGFEM (3.1), we first derive some preliminary results. In particular, we revisit some polynomial approximation and inverse estimates in the context of general polygonal/polyhedral elements.

We require the following assumptions on the mesh.

Assumption 4.1. There exists a positive constant $C_{F}$, independent of the mesh parameters, such that

$$
\max _{\kappa \in \mathcal{T}}(\operatorname{card}\{F \subset \Gamma: F \subset \partial \kappa\}) \leq C_{F} .
$$

Further, we require the existence of suitable coverings of the mesh, as specified below.

Definition 4.1. A covering $\mathcal{T}_{\sharp}=\{\mathcal{K}\}$ related to the polygonal/polyhedral mesh $\mathcal{T}$ is a set of shape-regular $d$-simplexes $\mathcal{K}$, such that for each $\kappa \in \mathcal{T}$, there exists a 
$\mathcal{K} \in \mathcal{T}_{\sharp}$ such that $\kappa \subset \mathcal{K}$. Given $\mathcal{T}_{\sharp}$, we denote by $\Omega_{\sharp}$ the covering domain given by $\Omega_{\sharp}:=\left(\cup_{\mathcal{K} \in \mathcal{T}_{\sharp}} \overline{\mathcal{K}}\right)^{\circ}$, where, for a closed set $D \subset \mathbb{R}^{d}, D^{\circ}$ denotes the interior of $D$.

Assumption 4.2. There exists a covering $\mathcal{T}_{\sharp}$ of $\mathcal{T}$ and a positive constant $\mathcal{O}_{\Omega}$, independent of the mesh parameters, such that

$$
\max _{\kappa \in \mathcal{T}} \mathcal{O}_{\kappa} \leq \mathcal{O}_{\Omega}
$$

where, for each $\kappa \in \mathcal{T}$,

$$
\mathcal{O}_{\kappa}:=\operatorname{card}\left\{\kappa^{\prime} \in \mathcal{T}: \kappa^{\prime} \cap \mathcal{K} \neq \emptyset, \mathcal{K} \in \mathcal{T}_{\sharp} \text { such that } \kappa \subset \mathcal{K}\right\} .
$$

As a consequence, we deduce that

$$
\operatorname{diam}(\mathcal{K}) \leq C_{\text {diam }} h_{\kappa},
$$

for each pair $\kappa \in \mathcal{T}, \mathcal{K} \in \mathcal{T}_{\sharp}$, with $\kappa \subset \mathcal{K}$, for a constant $C_{\text {diam }}>0$, uniformly with respect to the mesh size.

We note that mesh-regularity is assumed for the mesh covering $\mathcal{T}_{\sharp}$, and not for the mesh $\mathcal{T}$. Assumption 4.2 asserts that the amount of overlap present in the covering $\mathcal{T}_{\sharp}$ remains bounded as the computational mesh $\mathcal{T}$ is refined, i.e., as the diameter of the polygonal/polyhedral elements tends to zero.

Our $h p$-approximation results and inverse estimates for polygonal/polyhedral elements are based on considering $d$-dimensional simplexes, cf. above, where standard results can be applied.

Definition 4.2. For each element $\kappa$ in the computational mesh $\mathcal{T}$, we define the family $\mathcal{F}_{b}^{\kappa}$ of all possible $d$-dimensional simplexes contained in $\kappa$ and having at least one face in common with $\kappa$. The notation $\kappa_{b}^{F}$ will be used to indicate a simplex belonging to $\mathcal{F}_{b}^{\kappa}$ and sharing with $\kappa \in \mathcal{T}$ a given face $F$.

As far as approximation is concerned, the standard $h p$-approximation results, cf. Ref. 63, for example, are applicable by noting that each polygonal/polyhedral element is a subset of a $d$-simplex belonging to the covering $\mathcal{T}_{\sharp}$ and that the local finite element spaces consist of polynomials without the use of finite element mappings. With this in mind, we recall some standard $h p$-approximation results on $d$-simplexes from Ref. $17(d=2)$ and Ref. $56(d=3)$; see also Refs. 18, 35 for similar results.

Lemma 4.1. Let $\mathcal{K} \in \mathcal{T}_{\sharp}$ be a d-simplex, $d=2,3$, of diameter $h_{\mathcal{K}}$. Suppose further that $\left.v\right|_{\mathcal{K}} \in H^{k}(\mathcal{K})$, for some $k \geq 0$. Then, for $p \in \mathbb{N}$, there exists $\Pi_{p} v \in \mathcal{P}_{p}(\mathcal{K})$, such that

$$
\left\|v-\Pi_{p} v\right\|_{H^{q}(\mathcal{K})} \leq C \frac{h_{\mathcal{K}}^{s-q}}{p^{k-q}}\|v\|_{H^{k}(\mathcal{K})}, \quad k \geq 0,
$$

for $0 \leq q \leq k$, and

$$
\left\|v-\Pi_{p} v\right\|_{L^{\infty}(\mathcal{K})} \leq C \frac{h_{\mathcal{K}}^{s-d / 2}}{p^{k-d / 2}}\|v\|_{H^{k}(\mathcal{K})}, \quad k>d / 2 .
$$


Here, $s=\min \{p+1, k\}$ and $C$ is a positive constant that depends on the shaperegularity of $\mathcal{K}$, but is independent of $v, h_{\mathcal{K}}$, and $p$.

Functions defined on $\Omega$ can be extended to the covering domain $\Omega_{\sharp}$ based on the following standard extension operator.

Theorem 4.1. Let $\Omega$ be a domain with a Lipschitz boundary. Then there exists a linear extension operator $\mathcal{E}: H^{s}(\Omega) \rightarrow H^{s}\left(\mathbb{R}^{d}\right), s \in \mathbb{N}_{0}$, such that $\left.\mathcal{E} v\right|_{\Omega}=v$ and

$$
\|\mathcal{E} v\|_{H^{s}\left(\mathbb{R}^{d}\right)} \leq C\|v\|_{H^{s}(\Omega)}
$$

where $C$ is a positive constant depending only on $s$ and $\Omega$.

Proof. See Stein ${ }^{65}$.

Given the operator $\Pi_{p}$ defined in Lemma 4.1 and the extension operator $\mathcal{E}$ given in Theorem 4.1, we now proceed to define a suitable projection operator onto the finite element space $S_{\mathcal{T}}^{\mathbf{p}}$. To this end, for $v \in L^{2}(\Omega)$, we define $\tilde{\Pi} v \in S_{\mathcal{T}}^{\mathbf{p}}$ elementwise as follows: for each $\kappa \in \mathcal{T}$ and given the associated element $\mathcal{K} \in \mathcal{T}_{\sharp}$, such that $\kappa \subset \mathcal{K}$, cf. Definition 4.1, we write

$$
\left.\tilde{\Pi} v\right|_{\kappa}:=\left.\Pi_{p_{\kappa}}\left(\left.\mathcal{E} v\right|_{\mathcal{K}}\right)\right|_{\kappa},
$$

where $\Pi_{p_{\kappa}}: L^{2}(\mathcal{K}) \rightarrow \mathcal{P}_{p_{\kappa}}(\mathcal{K})$ as in Lemma 4.1 .

Lemma 4.2. Let $\kappa \in \mathcal{T}, F \subset \partial \kappa$ denote one of its faces, and $\mathcal{K} \in \mathcal{T}_{\sharp}$ denote the corresponding simplex such that $\kappa \subset \mathcal{K}$, cf. Definition 4.1. Suppose that $v \in L^{2}(\Omega)$ is such that $\left.\mathcal{E} v\right|_{\mathcal{K}} \in H^{k_{\kappa}}(\mathcal{K})$, for some $k \geq 0$. Then, given Assumption 4.2 is satisfied, the following bounds hold

$$
\|v-\tilde{\Pi} v\|_{H^{q}(\kappa)} \leq C \frac{h_{\kappa}^{s_{\kappa}-q}}{p_{\kappa}^{k_{\kappa}-q}}\|\mathcal{E} v\|_{H^{k_{\kappa}}(\mathcal{K})}, \quad k_{\kappa} \geq 0,
$$

for $0 \leq q \leq k_{\kappa}$, and

$$
\|v-\tilde{\Pi} v\|_{L^{2}(F)} \leq C|F|^{1 / 2} \frac{h_{\kappa}^{s_{\kappa}-d / 2}}{p_{\kappa}^{k_{\kappa}-1 / 2}} C_{m}\left(p_{\kappa}, \kappa, F\right)^{1 / 2}\|\mathcal{E} v\|_{H^{k_{\kappa}}(\mathcal{K})}, \quad k_{\kappa}>d / 2,
$$

where

$$
C_{m}\left(p_{\kappa}, \kappa, F\right)=\min \left\{\frac{h_{\kappa}^{d}}{\sup _{\kappa_{\mathrm{b}}^{F} \subset \kappa}\left|\kappa_{\mathrm{b}}^{F}\right|}, \frac{1}{p_{\kappa}^{1-d}}\right\} .
$$

Here, $s_{\kappa}=\min \left\{p_{\kappa}+1, k_{\kappa}\right\}$ and $C$ is a positive constant, that depends on the shaperegularity of $\mathcal{K}$, but is independent of $v, h_{\kappa}$, and $p_{\kappa}$.

Proof. We begin by observing that

$$
\|v-\tilde{\Pi} v\|_{H^{q}(\kappa)}=\left\|\mathcal{E} v-\Pi_{p_{\kappa}}(\mathcal{E} v)\right\|_{H^{q}(\kappa)} \leq\left\|\mathcal{E} v-\Pi_{p_{\kappa}}(\mathcal{E} v)\right\|_{H^{q}(\mathcal{K})} .
$$

Thereby, bound (4.4) follows immediately upon application of (4.1), noting Assumption 4.2 . 
To prove (4.5), we let $\kappa_{b}^{F} \in \mathcal{F}_{b}^{\kappa}$; applying a standard scaling argument with respect to $\kappa_{b}^{F}$, the multiplicative trace inequality, and (4.4), we obtain

$$
\begin{aligned}
\|v-\tilde{\Pi} v\|_{L^{2}(F)}^{2} \leq C|F| & \left(\frac{1}{\left|\kappa_{b}^{F}\right|}\|v-\tilde{\Pi} v\|_{L^{2}\left(\kappa_{b}^{F}\right)}^{2}\right. \\
& \left.+\left|\kappa_{b}\right|^{-1+1 / d}\|v-\tilde{\Pi} v\|_{L^{2}\left(\kappa_{b}^{F}\right)}\|\nabla(v-\tilde{\Pi} v)\|_{L^{2}\left(\kappa_{b}^{F}\right)}\right) \\
\leq C & \frac{|F|}{\left|\kappa_{b}^{F}\right|}\left(\frac{h_{\kappa}}{p_{\kappa}}+\left|\kappa_{b}^{F}\right|^{1 / d}\right) \frac{h_{\kappa}^{2 \kappa_{\kappa}-1}}{p_{\kappa}^{2 k_{\kappa}-1}}\|\mathcal{E} v\|_{H^{k_{\kappa}}(\mathcal{K})}^{2} .
\end{aligned}
$$

Given that $\left|\kappa_{b}^{F}\right| \leq h_{\kappa}^{d}$ and $\kappa_{b}^{F}$ is arbitrary, from (4.6) we conclude that

$$
\|v-\tilde{\Pi} v\|_{L^{2}(F)}^{2} \leq C \frac{|F|}{\sup _{\kappa_{b}^{F} \subset \kappa}\left|\kappa_{b}^{F}\right|} \frac{h_{\kappa}^{2 s_{\kappa}}}{p_{\kappa}^{2 k_{\kappa}-1}}\|\mathcal{E} v\|_{H^{k_{\kappa}(\mathcal{K})}}^{2} .
$$

On the other hand, we observe that

$$
\|v-\tilde{\Pi} v\|_{L^{2}(F)}^{2} \leq|F|\|v-\tilde{\Pi} v\|_{L^{\infty}(F)}^{2} ;
$$

employing the definition of the projection operator $\tilde{\Pi}$, cf. above, together with (4.2) and Assumption 4.2 gives

$$
\|v-\tilde{\Pi} v\|_{L^{2}(F)}^{2} \leq C|F| \frac{h_{\kappa}^{2 s_{\kappa}-d}}{p_{\kappa}^{2 k_{\kappa}-d}}\|\mathcal{E} v\|_{H^{k_{\kappa}}(\mathcal{K})}^{2} .
$$

Now (4.5) follows by taking the minimum between the bounds (4.7) and (4.8).

Remark 4.1. We note that (4.7) is valid for $k_{\kappa} \geq 1$, but we omitted this level of generality in the statement of Lemma 4.2 in the interest of simplicity of the presentation.

Classical inverse estimates, bounding a norm of a polynomial on an element face by a norm on the element itself, are an essential ingredient in the error analysis of DGFEMs. More specifically, the stability and a priori error analysis of standard $h p$-version DGFEMs makes use of inverse estimates of the following form

$$
\|v\|_{L^{2}(F)}^{2} \leq C_{\text {inv }} \frac{p^{2}|F|}{|\kappa|}\|v\|_{L^{2}(\kappa)}^{2},
$$

where $F$ denotes a face of the simplicial or quadrilateral/hexahedral element $\kappa$ and $v$ is a polynomial of degree $p$. Furthermore, $C_{\text {inv }}$ is a positive constant, which is independent of $v, p,|F|$, and $|\kappa|$.

It is possible to generalize the above inverse estimate (4.9) to the case when $\kappa$ is a general polygonal/polyhedral element, based on employing (overlapping) subtriangulations of $\kappa$. To this end, we recall the family of simplexes $\mathcal{F}_{b}^{\kappa}$ of Definition 4.2 and consider $\kappa_{b}^{F} \in \mathcal{F}_{b}^{\kappa}$. Then, for $v \in \mathcal{P}_{p}(\kappa)$, applying (4.9) on $\kappa_{b}^{F}$, we have

$$
\|v\|_{L^{2}(F)}^{2} \leq C_{\mathrm{inv}} \frac{p^{2}|F|}{\left|\kappa_{b}^{F}\right|}\|v\|_{L^{2}\left(\kappa_{b}^{F}\right)}^{2} \leq C_{\mathrm{inv}} \frac{p^{2}|F|}{\left|\kappa_{b}^{F}\right|}\|v\|_{L^{2}(\kappa)}^{2},
$$




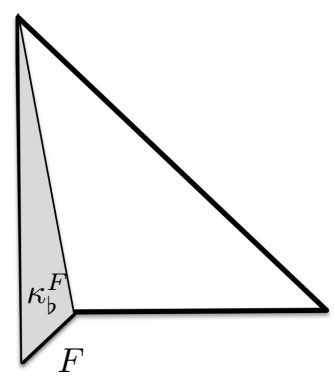

Fig. 1. Illustration of the quadrilateral in Example 4.1

where $C_{\text {inv }}$ is a positive contant, independent of $v,|F|,\left|\kappa_{b}^{F}\right|$, and $p$. Further, as in Lemma 4.2 , one can select $\kappa_{b}^{F}$ to have the largest possible measure $\left|\kappa_{b}^{F}\right|$, resulting in an inverse estimate of the form

$$
\|v\|_{L^{2}(F)}^{2} \leq C_{\mathrm{inv}} \frac{p^{2}|F|}{\sup _{\kappa_{b}^{F} \subset \kappa}\left|\kappa_{b}^{F}\right|}\|v\|_{L^{2}(\kappa)}^{2} .
$$

Apart from the obvious issue of requiring to solve a small optimization problem to evaluate accurately $\sup _{\kappa_{b}^{F} \subset \kappa}\left|\kappa_{b}^{F}\right|$, the inverse estimate (4.11) lacks sharpness in that it is not sensitive to the magnitude of the face measure relative to the measure of the polygonal element $\kappa$. For illustration, we consider the following example.

Example 4.1. We consider the domain $\kappa:=\left\{(x, y) \in \mathbb{R}^{2}: x>0, y>0, x+y<\right.$ $1\} \cup\left\{(x, y) \in \mathbb{R}^{2}: x>0, y \leq 0, x-y<\epsilon\right\}$, for some $\epsilon>0$; we refer to Figure 1 for an illustration. Given $v \in \mathcal{P}_{p}(\kappa)$, let $F:=\left\{(x, y) \in \mathbb{R}^{2}: x-y=\epsilon\right\}$, then upon application of (4.11), we deduce that

$$
\|v\|_{L^{2}(F)}^{2} \leq C_{\mathrm{inv}} \frac{\sqrt{2} p^{2} \epsilon}{\left|\kappa_{\mathrm{b}}^{F}\right|}\|v\|_{L^{2}(\kappa)}^{2},
$$

with $\kappa_{b}^{F}:=\left\{(x, y) \in \mathbb{R}^{2}: x>0, x+\epsilon y<\epsilon, x-y<\epsilon\right\}$. Thereby, given that $\left|\kappa_{b}^{F}\right|=\epsilon(1+\epsilon) / 2$, inequality (4.12) becomes

$$
\|v\|_{L^{2}(F)}^{2} \leq C_{\text {inv }} \frac{2 \sqrt{2} p^{2}}{1+\epsilon}\|v\|_{L^{2}(\kappa)}^{2} .
$$

Now as $\epsilon \rightarrow 0$, the left-hand side $\|v\|_{L^{2}(F)}^{2} \rightarrow 0$, whereas the right-hand side $\frac{2 \sqrt{2} p^{2}}{1+\epsilon}\|v\|_{L^{2}(\kappa)}^{2} \rightarrow 2 \sqrt{2} p^{2}\|v\|_{L^{2}(\kappa)}^{2} \neq 0$ in general.

This lack of sharpness in the inverse estimate (4.11), with respect to faces of degenerating (Hausdorff) measure, may lead to the definition of an excessively large penalization term when considering the stability of the IP DGFEM (3.1); this in turn, may result in ill conditioning of the underlying stiffness matrix. To overcome this issue, we proceed as follows: from the first inequality in (4.10), we have

$$
\|v\|_{L^{2}(F)}^{2} \leq C_{\text {inv }} p^{2}|F|\|v\|_{L^{\infty}\left(\kappa_{\mathrm{b}}^{F}\right)}^{2},
$$




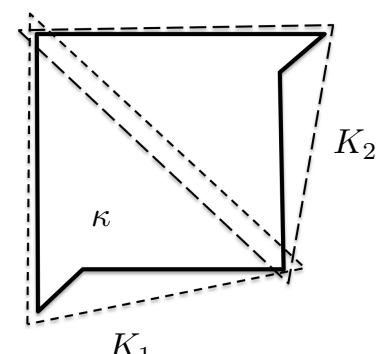

Fig. 2. Illustration of quadrilateral in Definition 4.3

using the trivial bound $\|v\|_{L^{2}\left(\kappa_{b}^{F}\right)}^{2} \leq\left|\kappa_{b}^{F}\right|\|v\|_{L^{\infty}\left(\kappa_{b}^{F}\right)}^{2}$. We now aim to provide an upper bound on $\|v\|_{L^{\infty}\left(\kappa_{b}^{F}\right)}^{2}$; to this end, we introduce the following definition.

Definition 4.3. Let $\tilde{\mathcal{T}}$ denote the subset of elements $\kappa, \kappa \in \mathcal{T}$, such that each $\kappa \in \tilde{\mathcal{T}}$ can be covered by at most $m_{\mathcal{T}}$ shape-regular simplexes $K_{i}, i=1, \ldots, m_{\mathcal{T}}$, such that

$$
\operatorname{dist}\left(\kappa, \partial K_{i}\right)<C_{a s} \operatorname{diam}\left(K_{i}\right) / p^{2},
$$

and

$$
\left|K_{i}\right| \geq c_{a s}|\kappa|,
$$

for all $i=1, \ldots, m_{\mathcal{T}}$, for some $m_{\mathcal{T}} \in \mathbb{N}$ and $C_{a s}, c_{a s}>0$, independent of $\kappa$ and $\mathcal{T}$.

We refer to Figure 2 for an illustration of a polygonal element $\kappa \in \tilde{\mathcal{T}}$ covered by two triangles $K_{1}$ and $K_{2}$. This definition allows for very general polygonal/polyhedral meshes to be included in the a priori error analysis given below: it is of relevance when the polygonal/polyhedral elements $\kappa \in \mathcal{T}$ contain faces/edges and faces whose (Hausdorff) measure is arbitrarily small. Note that, in particular, the polygon of Example 4.1 satisfies Definition 4.3, when $\epsilon<C_{a s} / p^{2}$ for some constant $C_{a s}>0$.

The motivation for Definition 4.3 comes from the following result, which is a straightforward generalization (via a standard scaling argument) of Lemma 3.7 in Ref. 41.

Lemma 4.3. Let $K$ be a shape-regular simplex. Then, for each $v \in \mathcal{P}_{p}(K)$, there exists a simplex $\hat{\kappa} \subset K$, having the same shape as $K$ and faces parallel to the faces of $K$, with $\operatorname{dist}(\partial \hat{\kappa}, \partial K)<C_{a s} \operatorname{diam}(K) / p^{2}$, for some constant $C_{a s}>0$, independent of $v, K$ and $p$, such that

$$
\|v\|_{L^{2}(\hat{\kappa})} \geq \frac{1}{2}\|v\|_{L^{2}(K)} .
$$

We are now ready to present an inverse estimate for a general polygon/polyhedron, which appears to be sharp with respect to degeneration of one or more of its faces. 
Lemma 4.4. Let $\kappa \in \mathcal{T}, F \subset \partial \kappa$ denote one of its faces, and $\tilde{\mathcal{T}}$ be defined as in Definition 4.3. Then, for each $v \in \mathcal{P}_{p}(\kappa)$, we have the inverse estimate

$$
\|v\|_{L^{2}(F)}^{2} \leq C_{\mathrm{INV}}(p, \kappa, F) \frac{p^{2}|F|}{|\kappa|}\|v\|_{L^{2}(\kappa)}^{2}
$$

with

$$
C_{\mathrm{INV}}(p, \kappa, F):=C_{\mathrm{inv}}\left\{\begin{array}{cl}
\min \left\{\frac{|\kappa|}{\sup _{\kappa_{b}^{F} \subset \kappa \mid}\left|\kappa_{b}^{F}\right|}, p^{2 d}\right\}, & \text { if } \kappa \in \tilde{\mathcal{T}}, \\
\frac{|\kappa|}{\sup _{\kappa_{b}^{F} \subset \kappa}\left|\kappa_{b}^{F}\right|}, & \text { if } \kappa \in \mathcal{T} \backslash \tilde{\mathcal{T}},
\end{array}\right.
$$

and $\kappa_{b}^{F} \in \mathcal{F}_{b}^{\kappa}$ as in Definition 4.2 and (4.11). Furthermore, $C_{\mathrm{inv}}$ is a positive constant, which if $\kappa \in \tilde{\mathcal{T}}$ depends on the shape regularity of the covering of $\kappa$ given in Definition 4.3, but is always independent of $|\kappa| / \sup _{\kappa_{b}^{F} \subset \kappa}\left|\kappa_{b}^{F}\right|$ (and, therefore, of $|F|), p$, and $v$.

Proof. For $\kappa \in \mathcal{T} \backslash \tilde{\mathcal{T}}$ the above inverse inequality follows immediately from (4.11). Let us now turn our attention to the case when $\kappa \in \tilde{\mathcal{T}}$. To this end, recalling Definition 4.3 , for $\kappa \in \tilde{\mathcal{T}}$, we have a covering of $\kappa$ by shape-regular simplexes $K_{j}$, $j=1, \ldots, m_{\mathcal{T}}$. Hence, for $\kappa_{\mathrm{b}}^{F} \in \mathcal{F}_{\mathrm{b}}^{\kappa}$, we have $\kappa_{\mathrm{b}}^{F} \subset \kappa \subset \cup_{j=1}^{m_{\mathcal{T}}} K_{j}$, with $\left|K_{j}\right| \geq c_{a s}|\kappa|$, for $\kappa_{b}^{F}$ as in (4.13).

We recall the standard inverse estimate

$$
\|v\|_{L^{\infty}\left(K_{j}\right)}^{2} \leq C_{\infty} \frac{p^{2 d}}{\left|K_{j}\right|}\|v\|_{L^{2}\left(K_{j}\right)}^{2},
$$

where $C_{\infty}$ is a positive constant, independent of $p,\left|K_{j}\right|$, and $v$; see, e.g., Schwab ${ }^{63}$. Employing (4.15) and Definition 4.3 again, we deduce that

$$
\begin{aligned}
\|v\|_{L^{\infty}\left(\kappa_{b}^{F}\right)}^{2} & \leq \sum_{j=1}^{m \mathcal{T}}\|v\|_{L^{\infty}\left(K_{j}\right)}^{2} \\
& \leq C_{\infty} p^{2 d} \sum_{j=1}^{m_{\mathcal{T}}} \frac{\|v\|_{L^{2}\left(K_{j}\right)}^{2}}{\left|K_{j}\right|} \\
& \leq \frac{C_{\infty}}{c_{a s}} \frac{p^{2 d}}{|\kappa|} \sum_{j=1}^{m_{\mathcal{T}}}\|v\|_{L^{2}\left(K_{j}\right)}^{2} .
\end{aligned}
$$

We now define $\hat{\kappa}_{j} \subset K_{j}$ to denote the simplex relative to $K_{j}$ as outlined in Lemma 4.3; thereby, exploiting Lemma 4.3 and Definition 4.3, gives

$$
\frac{1}{4}\|v\|_{L^{2}\left(K_{j}\right)}^{2} \leq\|v\|_{L^{2}\left(\hat{\kappa}_{j}\right)}^{2} \leq\|v\|_{L^{2}\left(K_{j} \cap \kappa\right)}^{2},
$$

since $\hat{\kappa}_{j} \subset \kappa$, and hence $\hat{\kappa}_{j} \subset K_{j} \cap \kappa \subset K_{j}$. Combining (4.16) and (4.17), we arrive at the inequality

$$
\|v\|_{L^{\infty}\left(\kappa_{b}^{F}\right)}^{2} \leq \frac{4 C_{\infty} m_{\mathcal{T}}}{c_{a s}} \frac{p^{2 d}}{|\kappa|}\|v\|_{L^{2}(\kappa)}^{2},
$$


upon observing the trivial relation $K_{j} \cap \kappa \subset \kappa$. Inserting (4.18) into (4.13) gives

$$
\|v\|_{L^{2}(F)}^{2} \leq C_{\mathrm{inv}, 2} \frac{p^{2 d+2}|F|}{|\kappa|}\|v\|_{L^{2}(\kappa)}^{2},
$$

with $C_{\text {inv }, 2}=4 C_{\text {inv }} C_{\infty} m_{\mathcal{T}} / c_{a s}$.

Taking the minimum between (4.11) and (4.19), we deduce the desired result, with a different constant, also denoted by $C_{\mathrm{inv}}>0$. Finally, we remark that $C_{\mathrm{inv}}$ is, indeed, independent of $|F|$ as is the ratio $|\kappa| / \sup _{\kappa_{b}^{F} \subset \kappa}\left|\kappa_{b}^{F}\right|$.

Remark 4.2. We stress that (4.14) is sharp with respect to the polynomial degree $p$. Indeed, as $p \rightarrow \infty$, the minimum in (4.14) will be equal to $|\kappa| / \sup _{\kappa_{\mathrm{b}}^{F} \subset \kappa}\left|\kappa_{b}^{F}\right|$; this will be of crucial importance in the error analysis below. Further, (4.14) is sensitive with respect to the measure of the face $F$ relative to that of the element $\kappa$. In three dimensions, face degeneration may occur when a face or one of its edges shrinks to a point (recall that here mesh faces are triangular). The inverse estimate (4.14) is sharp in both cases of face and edge degeneration. For instance, going back to the setting of Example 4.1, we can see that both the left- and right-hand sides of (4.14) degenerate at the same rate as $\epsilon \rightarrow 0$. In summary, the two cases in the constant of (4.14) serves the two extremes of one degenerating face, for fixed $p$, and enrichment of the polynomial order $p$, while the element $\kappa$ is kept fixed.

Remark 4.3. We point out that $1 / \sup _{\kappa_{b}^{F} \subset \kappa}\left|\kappa_{b}^{F}\right|$ can be estimated in practice by selecting a simplex with face $F$ and the remaining vertex given by any of the nonadjacent vertices to $F$ of the polygon/polyhedron $\kappa$.

\section{A priori error bound}

Following the work presented in Ref. 59 for the local DGFEM, the a priori error analysis of the IP DGFEM (3.1) is undertaken based on introducing an appropriate inconsistent formulation. To this end, we begin by defining suitable extensions of the forms $B$ and $\ell$. In particular, we define the bilinear form

$$
\begin{aligned}
\tilde{B}(w, v):= & \sum_{\kappa \in \mathcal{T}} \int_{\kappa} \nabla w \cdot \nabla v \mathrm{~d} \mathbf{x}-\int_{\Gamma \backslash \Gamma_{\mathrm{N}}}\left(\left\{\boldsymbol{\Pi}_{2}\left(\nabla_{h} w\right)\right\} \cdot \llbracket v \rrbracket+\left\{\left\{\boldsymbol{\Pi}_{2}\left(\nabla_{h} v\right)\right\} \cdot \llbracket w \rrbracket\right) \mathrm{d} s\right. \\
& +\int_{\Gamma \backslash \Gamma_{\mathrm{N}}} \sigma \llbracket w \rrbracket \cdot \llbracket v \rrbracket \mathrm{d} s,
\end{aligned}
$$

and linear functional

$$
\tilde{\ell}(v)=\int_{\Omega} f v \mathrm{~d} \mathbf{x}-\int_{\Gamma_{\mathrm{D}}} g_{\mathrm{D}}\left(\boldsymbol{\Pi}_{2}\left(\nabla_{h} v\right) \cdot \mathbf{n}-\sigma v\right) \mathrm{d} s+\int_{\Gamma_{\mathrm{N}}} g_{\mathrm{N}} v \mathrm{~d} s .
$$

Here, $\Pi_{2}:\left[L^{2}(\Omega)\right]^{d} \rightarrow\left[S_{\mathcal{T}}^{\mathbf{p}}\right]^{d}$ denotes the orthogonal $L^{2}$-projection onto the finite element space $\left[S_{\mathcal{T}}^{\mathbf{p}}\right]^{d}$. In this manner, the face integrals involving the terms $\left\{\left\{\boldsymbol{\Pi}_{2}\left(\nabla_{h} w\right)\right\},\left\{\left\{\boldsymbol{\Pi}_{2}\left(\nabla_{h} v\right)\right\}\right.\right.$ and $\boldsymbol{\Pi}_{2}\left(\nabla_{h} v\right)$ are well defined for all $v, w \in \mathcal{S}:=$ 
$H^{1}(\Omega)+S_{\mathcal{T}}^{\mathbf{p}}$, as these terms are now traces of elementwise polynomial functions. Moreover, it is evident that

$$
\tilde{B}(w, v)=B(w, v) \quad \text { for all } \quad w, v \in S_{\mathcal{T}}^{\mathbf{p}},
$$

and

$$
\tilde{\ell}(v)=\ell(v) \quad \text { for all } \quad v \in S_{\mathcal{T}}^{\mathbf{p}} .
$$

Thereby, $\tilde{B}(\cdot, \cdot)$ and $\tilde{\ell}(\cdot)$ are extensions of $B(\cdot, \cdot)$ and $\ell(\cdot)$ to $\mathcal{S} \times \mathcal{S}$ and $\mathcal{S}$, respectively. Hence, we may rewrite the discrete problem (3.1) in the following equivalent form: find $u_{h} \in S_{\mathcal{T}}^{\mathbf{p}}$ such that

$$
\tilde{B}\left(u_{h}, v_{h}\right)=\tilde{\ell}\left(v_{h}\right) \quad \forall v_{h} \in S_{\mathcal{T}}^{\mathbf{p}} .
$$

However, we point out that the IP DGFEM formulation (5.1) is no longer consistent due to the discrete nature of the $L^{2}$-projection operator $\boldsymbol{\Pi}_{2}$.

For the proceeding error analysis, we introduce the DG-norm $|\|\cdot \mid\|$ by

$$
\|w \mid\|:=\left(\sum_{\kappa \in \mathcal{T}} \int_{\kappa}|\nabla w|^{2} \mathrm{~d} \mathbf{x}+\int_{\Gamma \backslash \Gamma_{\mathrm{N}}} \sigma|\llbracket w \rrbracket|^{2} \mathrm{~d} s\right)^{1 / 2},
$$

for $w \in \mathcal{S}$ and $\sigma>0$.

With this notation, we establish the following coercivity and continuity properties of the bilinear form $\tilde{B}(\cdot, \cdot)$.

Lemma 5.1. Let $\sigma: \Gamma \backslash \Gamma_{N} \rightarrow \mathbb{R}_{+}$be defined facewise by

$$
\sigma(\mathbf{x}):= \begin{cases}C_{\sigma \in\left\{\kappa^{+}, \kappa^{-}\right\}}\left\{C_{\mathrm{INV}}\left(p_{\kappa}, \kappa, F\right) \frac{p_{\kappa}^{2}|F|}{|\kappa|}\right\}, & \mathbf{x} \in F \subset \Gamma_{i n t}, F=\partial \kappa^{+} \cap \partial \kappa^{-}, \\ C_{\sigma} C_{\mathrm{INV}}\left(p_{\kappa}, \kappa, F\right) \frac{p^{2}|F|}{|\kappa|}, & \mathbf{x} \in F \subset \Gamma_{D}, F=\partial \kappa \cap \Gamma_{D},\end{cases}
$$

with $C_{\sigma}>0$ large enough, depending on $C_{F}$, and independent of $p,|F|$, and $|\kappa|$. Then, given Assumption 4.1 holds, we have that

$$
\tilde{B}(v, v) \geq C_{\text {coer }}\|v\|^{2} \quad \text { for all } \quad v \in \mathcal{S},
$$

and

$$
\tilde{B}(w, v) \leq C_{\text {cont }}|\|w|\||\|v \mid\| \quad \text { for all } \quad w, v \in \mathcal{S},
$$

where $C_{\text {coer }}$ and $C_{\text {cont }}$ are positive constants, independent of the discretization parameters.

Proof. The proof of (5.3) follows a standard argument, though for completeness, we outline the key steps. For $v \in \mathcal{S}$, we have

$$
\begin{aligned}
\tilde{B}(v, v) & =\|v \mid\|^{2}-2 \int_{\Gamma \backslash \Gamma_{\mathrm{N}}}\left\{\left[\boldsymbol{\Pi}_{2}\left(\nabla_{h} v\right)\right\}\right\} \cdot \llbracket v \rrbracket \mathrm{d} s \\
& \equiv \mathrm{I}+\mathrm{II} .
\end{aligned}
$$


In order to bound term II, we first note that for $F \subset \Gamma_{\text {int }}$, we have

$$
\begin{aligned}
\int_{F}\left\{\left\{\Pi_{2}\left(\nabla_{h} v\right)\right\}\right\} \cdot \llbracket v \rrbracket \mathrm{d} s \leq & \| \sigma^{-1 / 2}\left\{\left\{\boldsymbol{\Pi}_{2}\left(\nabla_{h} v\right)\right\}\left\|_{L^{2}(F)}\right\| \sigma^{1 / 2} \llbracket v \rrbracket \|_{L^{2}(F)}\right. \\
\leq & \frac{1}{2}\left(\left\|\sigma^{-1 / 2} \Pi_{2}\left(\nabla v^{+}\right)\right\|_{L^{2}(F)}+\left\|\sigma^{-1 / 2} \Pi_{2}\left(\nabla v^{-}\right)\right\|_{L^{2}(F)}\right) \\
& \times\left\|\sigma^{1 / 2} \llbracket v \rrbracket\right\|_{L^{2}(F)} \\
\leq & \epsilon\left(\left\|\sigma^{-1 / 2} \Pi_{2}\left(\nabla v^{+}\right)\right\|_{L^{2}(F)}^{2}+\left\|\sigma^{-1 / 2} \Pi_{2}\left(\nabla v^{-}\right)\right\|_{L^{2}(F)}^{2}\right) \\
& +\frac{1}{8 \epsilon}\left\|\sigma^{1 / 2} \llbracket v \rrbracket\right\|_{L^{2}(F)}^{2} ;
\end{aligned}
$$

here, we have employed the Cauchy-Schwarz inequality, together with the arithmetic-geometric mean inequality. Employing the inverse inequality stated in Lemma 4.4, we deduce that

$$
\begin{aligned}
\int_{F}\left\{\left\{\Pi_{2}\left(\nabla_{h} v\right)\right\} \cdot \llbracket v \rrbracket \mathrm{d} s \leq\right. & \epsilon\left(C_{\mathrm{INV}}\left(p_{\kappa^{+}}, \kappa^{+}, F\right) \frac{p_{\kappa^{+}}^{2}|F|}{\left|\kappa^{+}\right|}\left\|\sigma^{-1 / 2} \Pi_{2}(\nabla v)\right\|_{L^{2}\left(\kappa^{+}\right)}^{2}\right. \\
& \left.+C_{\mathrm{INV}}\left(p_{\kappa^{-}}, \kappa^{-}, F\right) \frac{p_{\kappa^{-}}^{2}|F|}{\left|\kappa^{-}\right|}\left\|\sigma^{-1 / 2} \Pi_{2}(\nabla v)\right\|_{L^{2}\left(\kappa^{-}\right)}^{2}\right) \\
& +\frac{1}{8 \epsilon}\left\|\sigma^{1 / 2} \llbracket v \rrbracket\right\|_{L^{2}(F)}^{2} \\
\leq & \frac{\epsilon}{C_{\sigma}}\left(\|\nabla v\|_{L^{2}\left(\kappa^{+}\right)}^{2}+\|\nabla v\|_{L^{2}\left(\kappa^{-}\right)}^{2}\right)+\frac{1}{8 \epsilon}\left\|\sigma^{1 / 2} \llbracket v \rrbracket\right\|_{L^{2}(F)}^{2},
\end{aligned}
$$

where we have used the definition of the interior penalty parameter $\sigma$, cf. (5.2), together with the $L^{2}$-stability of the projector $\Pi_{2}$.

In an analogous fashion, for $F \subset \Gamma_{\mathrm{D}}$, we have that

$$
\int_{F}\left\{\left[\Pi_{2}\left(\nabla_{h} v\right)\right\}\right] \cdot \llbracket v \rrbracket \mathrm{d} s \leq \frac{\epsilon}{C_{\sigma}}\|\nabla v\|_{L^{2}\left(\kappa^{+}\right)}^{2}+\frac{1}{4 \epsilon}\left\|\sigma^{1 / 2} \llbracket v \rrbracket\right\|_{L^{2}(F)}^{2} .
$$

Thereby, exploiting Assumption 4.1 above, inserting (5.6) and (5.7) into (5.5) gives

$$
\tilde{B}(v, v) \geq\left(1-\frac{2 C_{F}}{C_{\sigma}} \epsilon\right) \sum_{\kappa \in \mathcal{T}}\|\nabla v\|_{L^{2}(\kappa)}^{2}+\left(1-\frac{1}{2 \epsilon}\right) \sum_{F \subset \Gamma \backslash \Gamma_{\mathrm{N}}}\left\|\sigma^{1 / 2} \llbracket v \rrbracket\right\|_{L^{2}(F)}^{2},
$$

and the bilinear form $\tilde{B}(\cdot, \cdot)$ is coercive over $\mathcal{S} \times \mathcal{S}$, assuming that $\epsilon>1 / 2$ and $C_{\sigma}>2 C_{F} \epsilon$.

The proof of continuity immediately follows based on employing analogous arguments.

From Lemma 5.1 and Strang's second lemma, see, for example, Refs. 66, 36, we immediately deduce the following abstract error bound.

Theorem 5.1. Assuming the hypotheses of Lemma 5.1 hold, we have that

$$
\left\|u-u_{h}\left|\left\|\leq\left(1+\frac{C_{\text {cont }}}{C_{\text {coer }}}\right) \inf _{v \in S_{\mathcal{T}}^{\mathrm{p}}} \mid\right\| u-v\|\|+\frac{1}{C_{\text {coer }}} \sup _{w \in S_{\mathcal{T}}^{\mathrm{p}}} \frac{\left|R_{h}(u, w)\right|}{\|w\|},\right.\right.
$$


where the residual $R_{h}$ is given by

$$
R_{h}(u, w)=\tilde{B}(u, w)-\tilde{\ell}(u, w) .
$$

With this bound, we now proceed to prove the main result of this section.

Theorem 5.2. Let $\Omega \subset \mathbb{R}^{d}, d=2,3$, be a bounded polyhedral domain, and let $\mathcal{T}=\{\kappa\}$ be a subdivision of $\Omega$ consisting of general polygonal/polyhedral elements satisfying Assumption 4.1. Further, $\mathcal{T}_{\sharp}=\{\mathcal{K}\}$ denotes the associated covering of $\Omega$ consisting of shape-regular d-simplexes as in Definition 4.1, satisfying Assumption 4.2. Let $u_{h} \in S_{\mathcal{T}}^{\mathbf{p}}$ be the IP DGFEM approximation to $u \in H^{1}(\Omega)$ defined by (3.1) with the discontinuity-penalization parameter given by (5.2), and suppose that $\left.u\right|_{\kappa} \in H^{k_{\kappa}}(\kappa), k_{\kappa}>1+d / 2$, for each $\kappa \in \mathcal{T}$, such that $\left.\mathcal{E} u\right|_{\mathcal{K}} \in H^{k_{\kappa}}(\mathcal{K})$, where $\mathcal{K} \in \mathcal{T}_{\sharp}$ with $\kappa \subset \mathcal{K}$. Then, the following bound holds:

$$
\left\|u-u_{h}\right\|^{2} \leq C \sum_{\kappa \in \mathcal{T}} \frac{h_{\kappa}^{2\left(s_{\kappa}-1\right)}}{p_{\kappa}^{2\left(k_{\kappa}-1\right)}}\left(1+\mathcal{G}_{\kappa}\left(F, C_{\mathrm{INV}}, C_{m}, p_{\kappa}\right)\right)\|\mathcal{E} u\|_{H^{k_{\kappa}}(\mathcal{K})}^{2},
$$

where

$$
\begin{aligned}
& \mathcal{G}_{\kappa}\left(F, C_{\mathrm{INV}}, C_{m}, p_{\kappa}\right)=p_{\kappa} h_{\kappa}^{-d} \sum_{F \subset \partial \kappa \backslash \Gamma_{N}} C_{m}\left(p_{\kappa}, \kappa, F\right) \sigma^{-1}|F| \\
& +p_{\kappa}^{2}|\kappa|^{-1} \sum_{F \subset \partial \kappa \backslash \Gamma_{N}} C_{\mathrm{INV}}\left(p_{\kappa}, \kappa, F\right) \sigma^{-1}|F|+h_{\kappa}^{-d+2} p_{\kappa}^{-1} \sum_{F \subset \partial \kappa \backslash \Gamma_{N}} C_{m}\left(p_{\kappa}, \kappa, F\right) \sigma|F|,
\end{aligned}
$$

with $s_{\kappa}=\min \left\{p_{\kappa}+1, k_{\kappa}\right\}$ and $p_{\kappa} \geq 1$. Here, $C$ is a positive constant which is independent of the discretization parameters.

Proof. From Theorem 5.1, we recall that the error satisfies the following bound

$$
\left|\left\|u-u_{h}\left|\left\|\leq\left(1+\frac{C_{\text {cont }}}{C_{\text {coer }}}\right) \inf _{v \in S_{\mathcal{T}}^{\mathrm{p}}}\left|\|u-v \mid\|+\frac{1}{C_{\text {coer }}} \sup _{w \in S_{\mathcal{T}}^{\mathrm{p}}} \frac{\left|R_{h}(u, w)\right|}{|\|w \mid\|},\right.\right.\right.\right.\right.
$$

where the residual $R_{h}(u, w)=\tilde{B}(u, w)-\tilde{\ell}(u, w)$. To bound the first term on the right-hand side of (5.8), we exploit the approximation results stated in Lemma 4.2, together with Assumptions 4.1; to this end, we deduce that

$$
\begin{aligned}
\inf _{v \in S_{\mathcal{T}}^{\mathrm{p}}} \mid \| & u-v\left|\left\|^{2} \leq \mid\right\| u-\tilde{\Pi} u \|^{2}\right. \\
\leq & C \sum_{\kappa \in \mathcal{T}} \frac{h_{\kappa}^{2\left(s_{\kappa}-1\right)}}{p_{\kappa}^{2\left(k_{\kappa}-1\right)}}\left(1+\frac{h_{\kappa}^{-d+2}}{p_{\kappa}} \sum_{F \subset \partial \kappa \backslash \Gamma_{\mathrm{N}}} C_{m}\left(p_{\kappa}, \kappa, F\right) \sigma|F|\right) \\
& \times\|\mathcal{E} u\|_{H^{k_{\kappa}}(\mathcal{K})}^{2} .
\end{aligned}
$$

We now proceed to derive a bound on the residual $R_{h}$; to this end, we first note that, upon application of integration by parts elementwise, together with the statement 
of the underlying partial differential (2.1), (2.2), we deduce that

$$
R_{h}\left(u, w_{h}\right)=\int_{\Gamma \backslash \Gamma_{\mathrm{N}}}\left\{\nabla \nabla u-\Pi_{2}(\nabla u)\right\} \cdot \llbracket w_{h} \rrbracket \mathrm{d} s .
$$

Employing the Cauchy Schwarz inequality gives

$$
\sup _{w_{h} \in S_{\mathcal{T}}^{\mathrm{p}}} \frac{\left|R_{h}\left(u, w_{h}\right)\right|}{||\left|w_{h}\right| \mid} \leq\left(\int_{\Gamma \backslash \Gamma_{\mathrm{N}}} \sigma^{-1} \mid\left\{\left.\left\{\nabla u-\Pi_{2}(\nabla u)\right\}\right|^{2} \mathrm{~d} s\right)^{1 / 2} .\right.
$$

Writing $\tilde{\boldsymbol{\Pi}}$ to denote the vector-valued generalization of the $h p$-projection operator $\tilde{\Pi}$ given in (4.3), defined componentwise, we note that

$$
\begin{aligned}
& \int_{\Gamma \backslash \Gamma_{\mathrm{N}}} \sigma^{-1} \mid\left\{\left.\left\{\nabla u-\boldsymbol{\Pi}_{2}(\nabla u)\right\}\right|^{2} \mathrm{~d} s\right. \\
& \quad \leq \int_{\Gamma \backslash \Gamma_{\mathrm{N}}} \sigma^{-1} \mid\left\{\left.\{\nabla u-\tilde{\boldsymbol{\Pi}}(\nabla u)\}\right|^{2} \mathrm{~d} s+\int_{\Gamma \backslash \Gamma_{\mathrm{N}}} \sigma^{-1} \mid\left\{\left.\left\{\Pi_{2}(\tilde{\boldsymbol{\Pi}}(\nabla u)-\nabla u)\right\}\right|^{2} \mathrm{~d} s\right.\right. \\
& \quad \equiv \mathrm{I}+\mathrm{II} .
\end{aligned}
$$

To bound Term I, we proceed as above; thereby, we have

$$
\mathrm{I} \leq C \sum_{\kappa \in \mathcal{T}} \frac{h_{\kappa}^{2\left(s_{\kappa}-1\right)}}{p_{\kappa}^{2\left(k_{\kappa}-1\right)}} \frac{h_{\kappa}^{-d}}{p_{\kappa}^{-1}}\left(\sum_{F \subset \partial \kappa \backslash \Gamma_{\mathrm{N}}} C_{m}\left(p_{\kappa}, \kappa, F\right) \sigma^{-1}|F|\right)\|\mathcal{E} u\|_{H^{k_{\kappa}}(\mathcal{K})}^{2} .
$$

Exploiting the inverse inequality stated in Lemma 4.4 , the $L^{2}$-stability of the projector $\boldsymbol{\Pi}_{2}$, and the approximation results stated in Lemma 4.2 Term II may be bounded as follows

$$
\mathrm{II} \leq C \sum_{\kappa \in \mathcal{T}} \frac{h_{\kappa}^{2\left(s_{\kappa}-1\right)}}{p_{\kappa}^{2\left(k_{\kappa}-1\right)}} \frac{|\kappa|^{-1}}{p_{\kappa}^{-2}}\left(\sum_{F \subset \partial \kappa \backslash \Gamma_{\mathrm{N}}} C_{\mathrm{INV}}\left(p_{\kappa}, \kappa, F\right) \sigma^{-1}|F|\right)\|\mathcal{E} u\|_{H^{k_{\kappa}}(\mathcal{K})}^{2} .
$$

Hence, (5.10) may be bounded as follows:

$$
\begin{aligned}
& \sup _{w_{h} \in S_{\mathcal{T}}^{\mathrm{p}}} \frac{\left|R_{h}\left(u, w_{h}\right)\right|}{||\left|w_{h}\right| \|} \\
& \leq C\left(\sum _ { \kappa \in \mathcal { T } } \frac { h _ { \kappa } ^ { 2 ( s _ { \kappa } - 1 ) } } { p _ { \kappa } ^ { 2 ( k _ { \kappa } - 1 ) } } \left(\frac{h_{\kappa}^{-d}}{p_{\kappa}^{-1}} \sum_{F \subset \partial \kappa \backslash \Gamma_{\mathrm{N}}} C_{m}\left(p_{\kappa}, \kappa, F\right) \sigma^{-1}|F|\right.\right. \\
&\left.\left.\quad+\frac{|\kappa|^{-1}}{p_{\kappa}^{-2}} \sum_{F \subset \partial \kappa \backslash \Gamma_{\mathrm{N}}} C_{\mathrm{INV}}\left(p_{\kappa}, \kappa, F\right) \sigma^{-1}|F|\right)\|\mathcal{E} u\|_{H^{k_{\kappa}}(\mathcal{K})}^{2}\right)^{1 / 2} .
\end{aligned}
$$

Finally, substituting (5.9) and (5.12) into (5.8), we deduce the statement of the theorem.

Remark 5.1. We note that the a priori bound stated in Theorem 5.2 for the IP DGFEM (3.1) holds without the need to impose any assumptions concerning 
the relative sizes of the $(d-1)$-dimensional faces $F, F \subset \partial \kappa$, of a given polygonal/polyhedral element $\kappa \in \mathcal{T}$. Recall that for $d=3$, a face $F \subset \partial \kappa, \kappa \in \mathcal{T}$, is a triangle belonging to an elemental polygonal interface; the interior penalty parameter $\sigma$ is constant on each such triangle, rather than the whole polygonal interface. This way, a $(d-2)$-dimensional edge of an elemental polygonal interface is also allowed to degenerate, while the $(d-1)$-dimensional measure of the interface can remain positive. This remarkable property highlights the advantages of the interface subdivision into triangles, which may have appeared to be somewhat arbitrary in the first instance.

Remark 5.2. As discussed above, for $d=3$, the penalty parameter $\sigma$ will, in general, vary on each triangular face of a polygonal interface. This is in contrast with standard IP DGFEM error analyses, whereby a constant penalty is chosen on each element interface (even when hanging nodes/edges are allowed, cf., e.g., 49). Allowing for a piecewise penalty parameter on each interface leads to a potentially "finer" choice of the penalization, by eliminating the incorporation of theoretically relevant (but impractical) quantities such as, local $h$ or $p$ quasi-uniformity or regularity of hanging nodes, into the penalisation constant. Moreover, piecewise constant penalty parameters on the constituent triangular faces of polygonal interfaces for $d=3$, are also practical in terms of implementation. Indeed, quadrature rules on such element interfaces are typically computed in a piecewise fashion anyway.

Remark 5.3. Assuming standard local quasi-uniformity assumptions, (along with quasi-uniformity of the triangulation of each polygonal element interface for $d=3$ ), it would be possible to choose a constant penalisation parameter on each polygonal interface, thereby reverting to the "standard" IP DGFEM setting. Theorem 5.2 could be easily modified to this effect also, after summation of the interface triangular penalty contributions.

Remark 5.4. For uniform orders $p_{\kappa}=p \geq 1, h=\max _{\kappa \in \mathcal{T}} h_{\kappa}, s_{\kappa}=s, s=$ $\min \{p+1, k\}, k>1+d / 2$, we point out that, under the assumption that the diameter of the faces of each element $\kappa \in \mathcal{T}$ are of comparable size to the diameter of the corresponding element, i.e., $\operatorname{diam}(F) \sim h_{\kappa}, F \subset \partial \kappa, \kappa \in \mathcal{T}$, so that $|F| \sim h_{\kappa}^{(d-1)}$, we get the bound

$$
\left\|u-u_{h} \mid\right\| \leq C \frac{h^{s-1}}{p^{k-3 / 2}}\|u\|_{H^{k}(\Omega)} .
$$

Here, we have employed Theorem 4.1, together with Assumption 4.2, assuming that for such element domains $C_{\mathrm{INV}}\left(p_{\kappa}, F\right)=\mathcal{O}(1)$ and $C_{m}\left(p_{\kappa}, F\right)=\mathcal{O}(1)$ uniformly for each face $F \subset \partial \kappa \backslash \Gamma_{\mathrm{N}}$ for all $\kappa \in \mathcal{T}$. This bound is optimal in $h$ and suboptimal in $p$ by $p^{1 / 2}$. This error estimate coincides with the bounds derived in Refs. 49, 62, for example, for IP DGFEMs defined on standard element domains. 


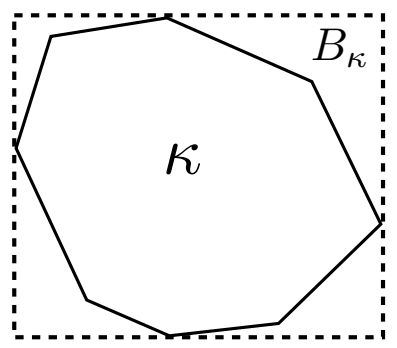

Fig. 3. Bounding box $B_{\kappa}$ of an element $\kappa \in \mathcal{T}$.

\section{Implementation issues}

In this section we briefly outline some of the implementation aspects of the proposed IP DGFEM, cf. (3.1).

\subsection{Construction of the finite element basis functions on general polygons/polyhedra}

The finite element space $S_{\mathcal{T}}^{\mathbf{p}}$ may be constructed in a number of different ways. In the case when the computational mesh $\mathcal{T}$ consists of standard affine element domains (simplexes, parallelograms, etc), standard polynomial bases on reference elements may simply be mapped from the reference frame to the physical element; indeed, this is the standard approach used within most finite element software packages. An alternative approach, which directly works on the physical element $\kappa \in \mathcal{T}$, without the need to refer to a given reference frame, has been proposed in the recent article by Bassi et $a l .{ }^{22}$; here, the elemental basis functions are constructed based on employing a Gram-Schmidt orthogonalization process applied to a given set of polynomial functions defined on $\kappa$. In this way, general polygonal/polyhedral elements may be treated in a simple fashion; indeed, Ref. 22 considers the application of this technique to general meshes consisting of agglomerated elements.

Here, we introduce an alternative approach based on employing polynomial spaces defined over the bounding box of each element; cf. Ref. 42. More precisely, given an element $\kappa \in \mathcal{T}$, we first construct the Cartesian bounding box $B_{\kappa}$, such that $\bar{\kappa} \subseteq \bar{B}_{\kappa}$, cf. Figure 3 . On the bounding box $B_{\kappa}$ we may define a standard polynomial space $\mathcal{P}_{p_{\kappa}}\left(B_{\kappa}\right)$ spanned by a set of basis functions $\left\{\phi_{i, \kappa}\right\}, i=1, \ldots, \operatorname{dim}\left(\mathcal{P}_{p_{\kappa}}\left(B_{\kappa}\right)\right)$. With this in mind, we employ tensor-product (scaled) Legendre polynomials; indeed, writing $I=(-1,1)$, we denote the family of $L^{2}(I)$-orthogonal (Legendre) polynomials by $\left\{L_{i}(x)\right\}_{i=0}^{\infty}$. Thereby, given a general interval $I_{b}=\left(x_{1}, x_{2}\right)$, the corresponding scaled Legendre polynomials may be defined by

$$
L_{i}^{[b]}(x)=\left(1 / h_{b}\right)^{1 / 2} L_{i}\left(\left(x-m_{b}\right) / h_{b}\right),
$$

where $h_{b}=\left(x_{2}-x_{1}\right) / 2$ and $m_{b}=\left(x_{1}+x_{2}\right) / 2$. With this notation, a polynomial basis on $B_{\kappa}$ may be defined as follows: writing $B_{\kappa}=I_{1} \times I_{2} \times \cdots \times I_{d}$, where $I_{j}$, 
$j=1, \ldots, d$, denotes a one-dimensional interval, the space of polynomials $\mathcal{P}_{p_{\kappa}}\left(B_{\kappa}\right)$ of total degree $p_{k}$ over $B_{\kappa}$ is given by

$$
\mathcal{P}_{p_{\kappa}}\left(B_{\kappa}\right)=\operatorname{span}\left\{\phi_{i, \kappa}\right\}_{i=1}^{\operatorname{dim}\left(\mathcal{P}_{p_{\kappa}}\left(B_{\kappa}\right)\right)},
$$

where

$\phi_{i, \kappa}(\mathbf{x})=L_{i_{1}}^{[1]}\left(x_{1}\right) L_{i_{2}}^{[2]}\left(x_{2}\right) \cdots L_{i_{d}}^{[d]}\left(x_{d}\right), \quad i_{1}+i_{2}+\ldots+i_{d} \leq p_{\kappa}, i_{k} \geq 0, k=1, \ldots, d$, and $\mathbf{x}=\left(x_{1}, x_{2}, \ldots, x_{d}\right)$. Thereby, the polynomial basis over the general polygonal/polyhedral element $\kappa$ may be defined by simply restricting the support of $\left\{\phi_{i, \kappa}\right\}, i=1, \ldots, \operatorname{dim}\left(\mathcal{P}_{p_{\kappa}}\left(B_{\kappa}\right)\right)$ to $\kappa$; i.e., the polynomial basis defined over $\kappa$ is given by $\left\{\left.\phi_{i, \kappa}\right|_{\kappa}\right\}, i=1, \ldots, \operatorname{dim}\left(\mathcal{P}_{p_{\kappa}}\left(B_{\kappa}\right)\right)$.

\subsection{Quadrature rules}

As in Ref. 51, quadrature over general polygonal/polyhedral element domains is undertaken based on first constructing a sub-triangulation, followed by the exploitation of standard integration schemes, cf. Ref. 64, for example. Thereby, given $\kappa \in \mathcal{T}$, we first construct a non-overlapping sub-triangulation $\kappa_{\mathcal{S}}=\left\{\tau_{\kappa}\right\}$ consisting of simplicial elements. As an example, if we consider the first term arising in the bilinear form $B(\cdot, \cdot)$, restricted to $\kappa$, then we compute

$$
\begin{aligned}
\int_{\kappa} \nabla w \cdot \nabla v \mathrm{~d} \mathbf{x} & =\sum_{\tau_{\kappa} \in \kappa_{\mathcal{S}}} \int_{\tau_{\kappa}} \nabla w \cdot \nabla v \mathrm{~d} \mathbf{x} \\
& \approx \sum_{\tau_{\kappa} \in \kappa_{\mathcal{S}}} \sum_{i=1}^{q} \nabla w\left(F_{\kappa}\left(\boldsymbol{\xi}_{i}\right)\right) \cdot \nabla v\left(F_{\kappa}\left(\boldsymbol{\xi}_{i}\right)\right) \operatorname{det}\left(J_{F_{\kappa}}\left(\boldsymbol{\xi}_{i}\right)\right) w_{i}
\end{aligned}
$$

where $F_{\kappa}: \hat{\kappa} \rightarrow \tau_{\kappa}$ is the mapping from the reference element (simplex) $\hat{\kappa}$ to $\tau_{\kappa}$, with Jacobi matrix $J_{F_{\kappa}}$, and $\left(\boldsymbol{\xi}_{i}, w_{i}\right)_{i=1}^{q}$ denotes the quadrature rule defined on $\hat{\kappa}$. We point out that the gradient operators are not transformed, as would be the case if the element $\kappa$ was mapped to a reference frame.

We point out that alternative integration methods which do not require a subtriangulation of the underlying polygonal/polyhedral element have recently been considered in Refs. 57, 24, 27. For related work, we refer to Refs. 22, 55, and the references cited therein.

\section{Numerical examples}

In this section we present a series of computational examples to numerically investigate the asymptotic convergence behaviour of the proposed IP DGFEM on general meshes consisting of polygonal elements. Throughout this section the IP DGFEM solution $u_{h}$ defined by $(3.1)$ is computed with the constant $C_{\sigma}$ appearing in the interior penalty parameter $\sigma$ defined in Lemma 5.1 equal to 10 . All the numerical examples presented in this section have been computed using the AptoFEM package (www.aptofem.com); here, the resulting system of linear equations is solved based 
December $\quad 2, \quad 2013 \quad 17: 11 \quad$ WSPC/INSTRUCTION $\quad$ FILE hp-DG' polygonal'elements vi3

on employing the Multifrontal Massively Parallel Solver (MUMPS), see Refs. 1, 2, 3.

\subsection{Example 1}

In this first example, we investigate the computational efficiency of employing the IP DGFEM on standard tensor-product elements (quadrilaterals in 2D and hexahedra in $3 \mathrm{D}$ ) employing local polynomial bases consisting of either $\mathcal{P}_{p}$ or $\mathcal{Q}_{p}$ polynomials; in the following figures, these schemes will be denoted by $\operatorname{DGFEM}(\mathrm{P})$ and $\operatorname{DGFEM}(\mathrm{Q})$, respectively. Moreover, we shall compare both IP DGFEM approaches with the standard continuous Galerkin finite element method (CGFEM).

Firstly, we consider the following two-dimensional problem: let $\Omega=(0,1)^{2}$ and select $f=2 \pi^{2} \sin (\pi x) \sin (\pi y)$, so that the analytical solution to (2.1) is given by $u=\sin (\pi x) \sin (\pi y)$. In Figure 4 we investigate the convergence behaviour of the three schemes, namely DGFEM(P), DGFEM(Q), and CGFEM, under $p$-refinement for fixed $h$. Here, uniform square meshes consisting of 16, 64, and 256 elements are employed; for each mesh, we plot both the $L^{2}(\Omega)$ norm and $H^{1}(\Omega)$ seminorm of the error, against the square root of the number of degrees of freedom in the underlying finite element space, as the polynomial degree $p$ is uniformly increased. Here, we clearly observe exponential convergence of all three methods, in the sense that, on the linear-log scale, the convergence plots become straight lines as $p$ is increased. Moreover, we observe that the convergence lines for CGFEM and DGFEM(Q) are roughly parallel, with the former method being more efficient, in the sense that, for a given number of degrees of freedom (dof), the error measured with respect to both the $L^{2}(\Omega)$ norm and $H^{1}(\Omega)$ seminorm is less than the corresponding quantity computed for $\operatorname{DGFEM}(\mathrm{Q})$. However, one important observation is that, for each mesh, the slope of the convergence line for $\operatorname{DGFEM}(\mathrm{P})$, i.e., the IP DGFEM employing local $\mathcal{P}_{p}$ polynomial bases, is actually steeper than the corresponding convergence line when local polynomial bases consisting of tensor-product $\mathcal{Q}_{p}$ polynomials are employed. Indeed, while for moderate $p$, we observe that the CGFEM method is more efficient than $\operatorname{DGFEM}(\mathrm{P})$, as the polynomial degree is increased, the convergence line for $\operatorname{DGFEM}(\mathrm{P})$ crosses the corresponding line for CGFEM, at least on the coarser meshes.

To investigate this behaviour further, we now consider the three-dimensional variant of the above problem. To this end, we let $\Omega=(0,1)^{3}$ and select $f=$ $3 \pi^{2} \sin (\pi x) \sin (\pi y) \sin (\pi z)$, so that the analytical solution to $(2.1)$ is given by $u=$ $\sin (\pi x) \sin (\pi y) \sin (\pi z)$. In Figure 5 we consider the convergence of the $\operatorname{DGFEM}(\mathrm{P})$, $\operatorname{DGFEM}(\mathrm{Q})$, and CGFEM schemes under $p$-refinement on uniform hexahedral meshes consisting of 64,512 , and 4096 elements. As in the two-dimensional setting, we again observe that the convergence lines for both CGFEM and DGFEM(Q) are roughly parallel, with, again, the former method being more efficient in terms of leading to a smaller error for a given number of degrees of freedom. Moreover, the slope of convergence line for the $\operatorname{DGFEM}(\mathrm{P})$ scheme is not only steeper than 
December 2
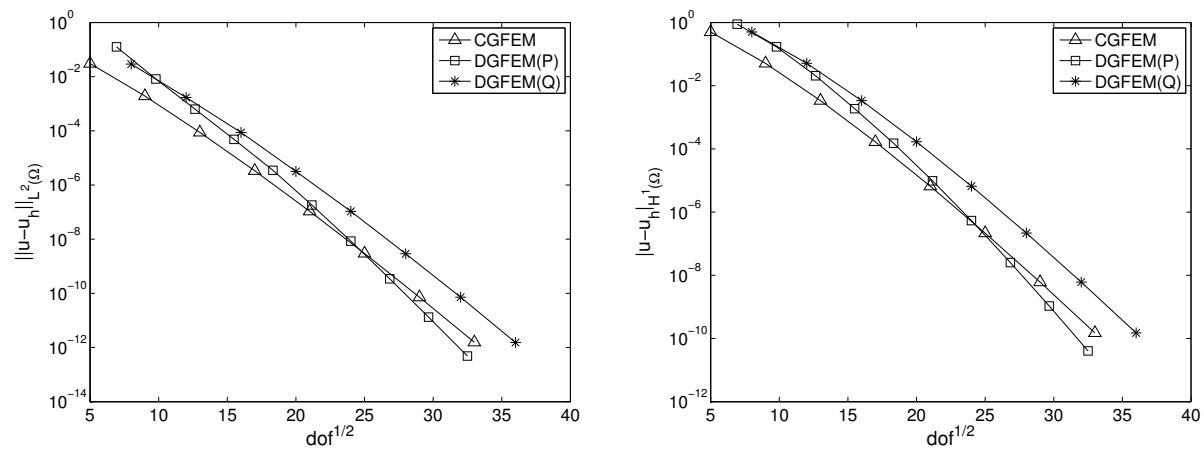

(a)
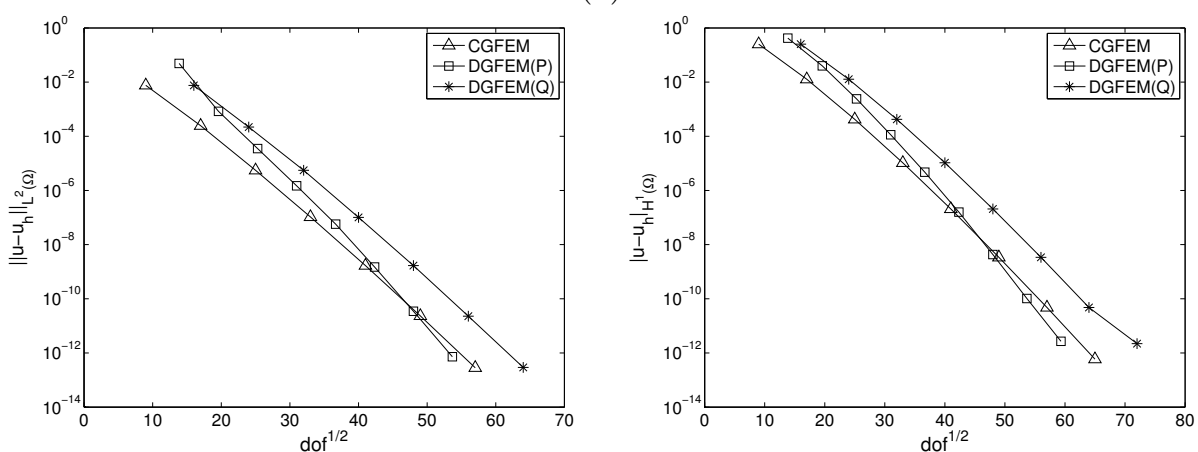

(b)
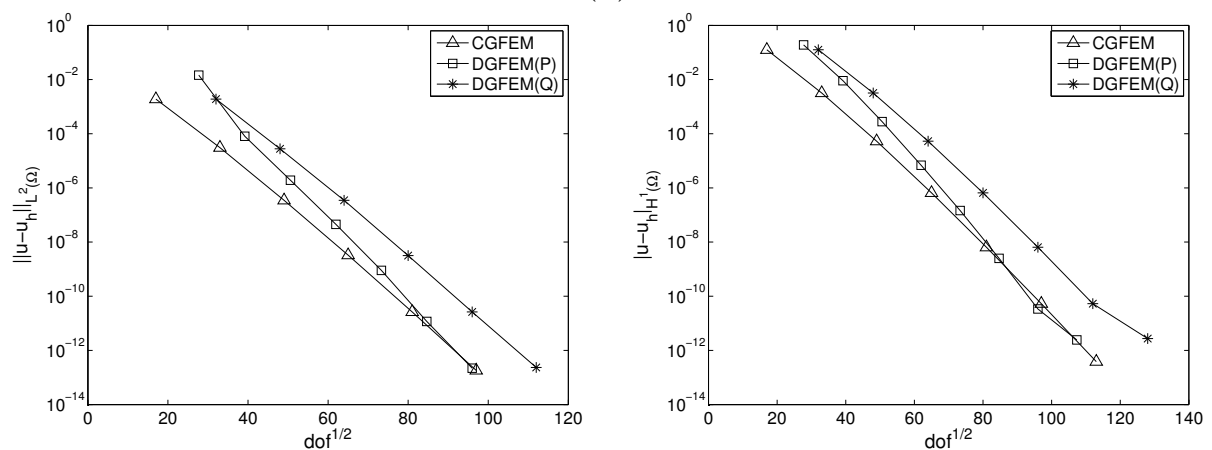

(c)

Fig. 4. Example 1. Comparison between IP DGFEM exploiting local $\mathcal{Q}_{p}$ and $\mathcal{P}_{p}$ polynomial spaces with CGFEM under $p$-refinement on uniform meshes consisting of square elements on $(0,1)^{2}(2 \mathrm{D})$. Left: $\left\|u-u_{h}\right\|_{L^{2}(\Omega)}$; Right: $\left|u-u_{h}\right|_{H^{1}(\Omega)}$; (a) $4 \times 4$ mesh; (b) $8 \times 8$ mesh; (c) $16 \times 16$ mesh.

the corresponding line for $\operatorname{DGFEM}(\mathrm{Q})$, but also that the cross-over point between $\operatorname{DGFEM}(\mathrm{P})$ becoming more efficient than CGFEM occurs much sooner. 
December

2
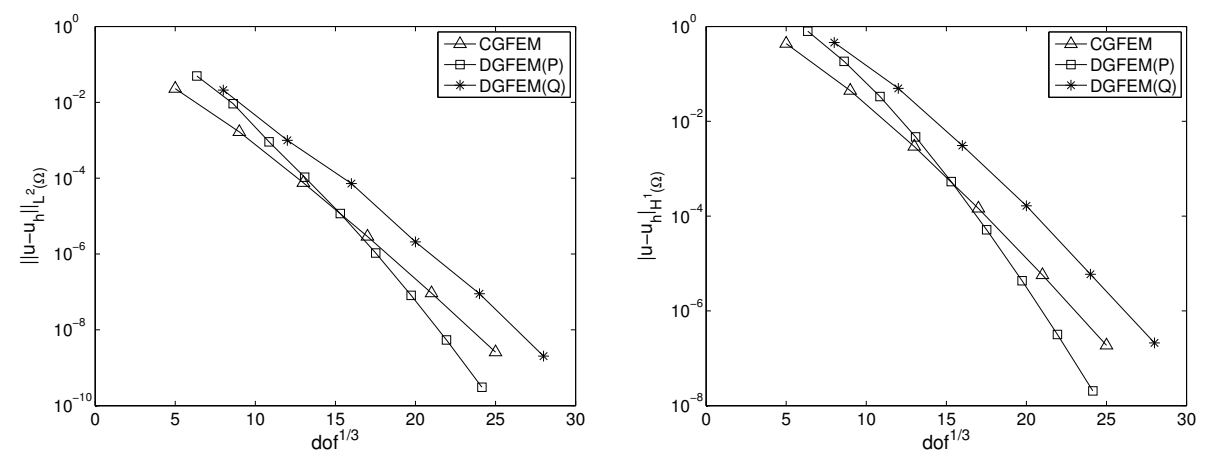

(a)
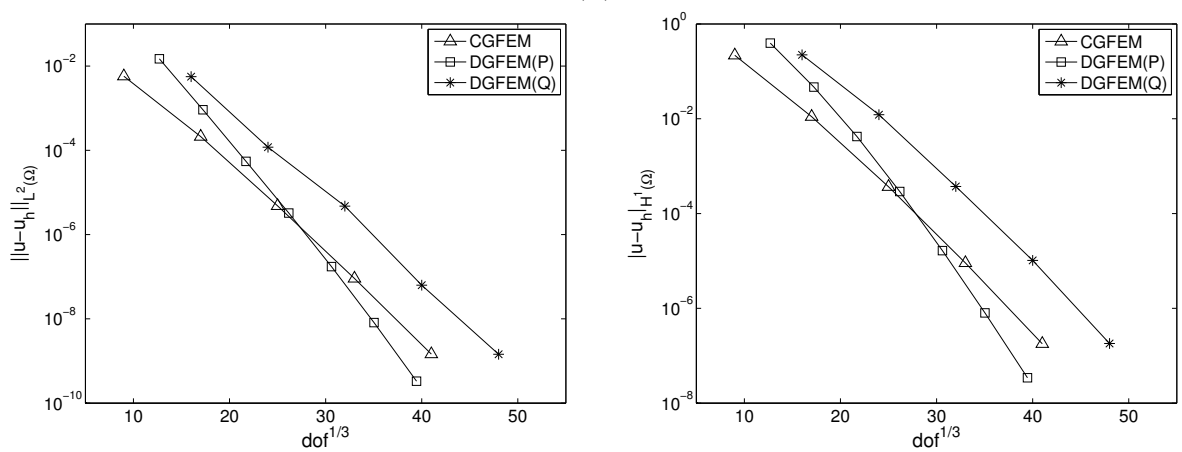

(b)
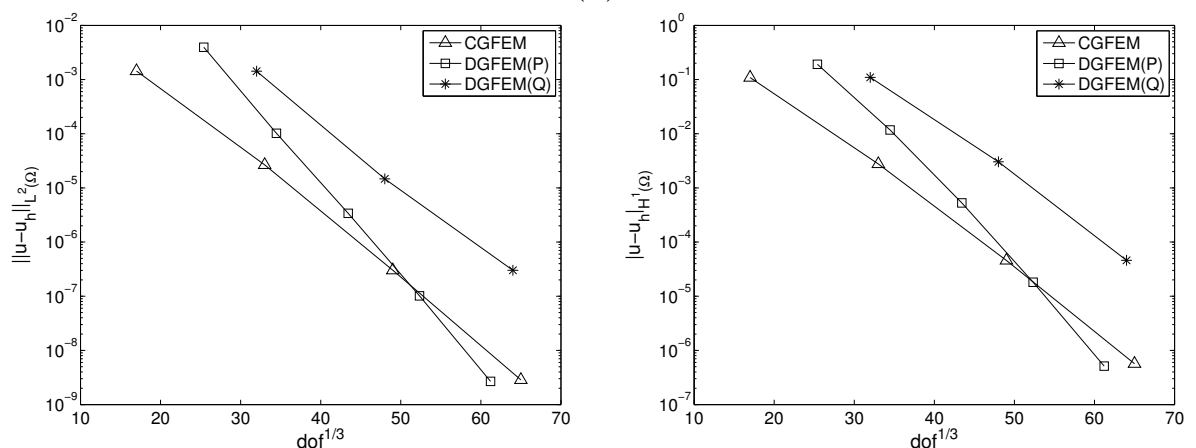

(c)

Fig. 5. Example 1. Comparison between IP DGFEM exploiting local $\mathcal{Q}_{p}$ and $\mathcal{P}_{p}$ polynomial spaces with CGFEM under $p$-refinement on uniform meshes consisting of hexahedral elements on $(0,1)^{3}$ (3D). Left: $\left\|u-u_{h}\right\|_{L^{2}(\Omega)}$; Right: $\left|u-u_{h}\right|_{H^{1}(\Omega)}$; (a) $4 \times 4 \times 4$ mesh; (b) $8 \times 8 \times 8$ mesh; (c) $16 \times 16 \times 16$ mesh.

\subsection{Example 2}

Following on from the previous numerical example, here we investigate the convergence behaviour of the $\operatorname{DGFEM}(\mathrm{P})$ and $\operatorname{DGFEM}(\mathrm{Q})$ approaches for a non-smooth 


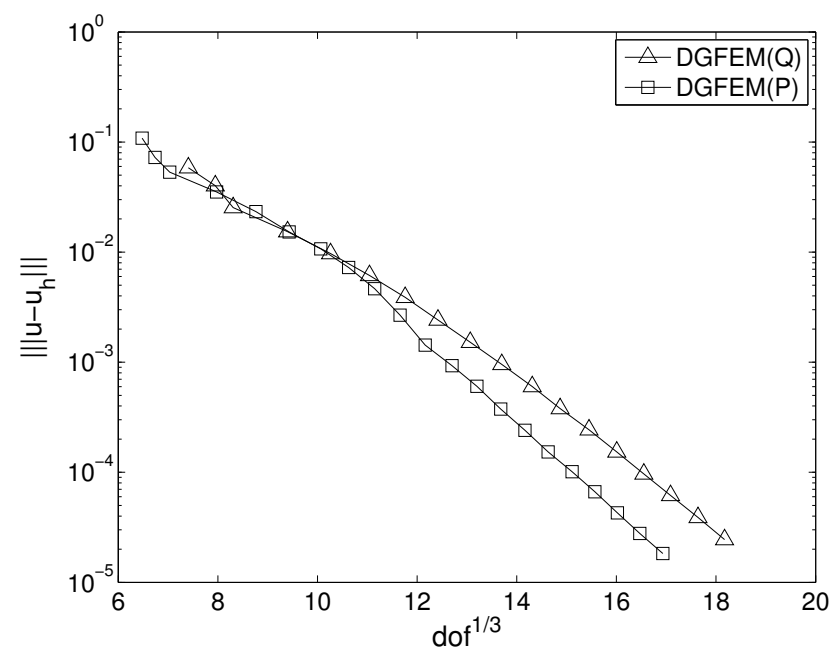

Fig. 6. Example 2. Comparison between IP DGFEM exploiting local $\mathcal{Q}_{p}$ and $\mathcal{P}_{p}$ polynomial spaces based on employing adaptive $h p$-refinement.

problem on $h p$-adaptively refined computational meshes. To this end, we let $\Omega$ be the L-shaped domain $(-1,1)^{2} \backslash[0,1) \times(-1,0]$, and select $f=0$. Then, writing $(r, \varphi)$ to denote the system of polar coordinates, we impose an appropriate inhomogeneous boundary condition for $u$ so that

$$
u=r^{2 / 3} \sin (2 \varphi / 3)
$$

cf. Ref. 71 . We note that $u$ is analytic in $\bar{\Omega} \backslash\{\mathbf{0}\}$, but $\nabla u$ is singular at the origin; indeed, here $u \notin H^{2}(\Omega)$. This example reflects the typical (singular) behaviour that solutions of elliptic boundary value problems exhibit in the vicinity of reentrant corners in the computational domain.

The underlying $h p$-adaptive algorithm exploited within this section is based on employing the residual-based a posteriori error indicators proposed in Ref. 47. More precisely, the $h p$-adaptive meshes are constructed by first marking the elements for refinement/derefinement according to the size of these local error indicators; this is done by employing the fixed fraction strategy, with refinement and derefinement fractions set to $25 \%$ and $10 \%$, respectively. The decision to perform local $h / p$ refinement/derefinement is based on employing the analyticity testing algorithm outlined in Ref. 50.

In Figure 6 we present a comparison of the actual error, measured in terms of the DG-norm $\|\cdot \mid\|$, for both $\operatorname{DGFEM}(\mathrm{P})$ and $\operatorname{DGFEM}(\mathrm{Q})$, versus the third root of the number of degrees of freedom in the underlying finite element space on a linear-log scale, for the sequence of meshes generated by the above $h p$-adaptive algorithm. For the initial refinement steps, we observe that the error in both the $\operatorname{DGFEM}(\mathrm{P})$ and DGFEM(Q) schemes is roughly comparable, for a given number of degrees of 
freedom. However, as the adaptive algorithm proceeds, and further $p$-enrichment is undertaken, we observe that the convergence line for $\operatorname{DGFEM}(\mathrm{P})$ becomes steeper than the corresponding line for DGFEM(Q), cf. the previous example; consequently, $\operatorname{DGFEM}(\mathrm{P})$ becomes more efficient than $\operatorname{DGFEM}(\mathrm{Q})$, in the sense that, for a given number of degrees of freedom, the error employing the former method is smaller than the corresponding quantity computed for $\operatorname{DGFEM}(\mathrm{Q})$.

\subsection{Example 3}

In this final example, we now turn our attention to investigate the asymptotic behaviour of the proposed IP DGFEM (DGFEM(P) using the previous notation) on a sequence of successively finer polygonal and square meshes for different values of the polynomial degree $p$; we point out that in both cases we employ local spaces consisting of polynomials of degree at most $p$ on each element $\kappa$ in the mesh $\mathcal{T}$. The polygonal meshes are generated using the general-purpose mesh generator PolyMesher, cf. Ref. 69. Here, we employ two types of meshes generated by PolyMesher: irregular meshes (without any mesh smoothing applied), whose elements possess faces of varying size (referred to as Polygonal Elements I), and regular meshes, generated by employing a number of smoothing steps within the mesh generator (referred to as Polygonal Elements II). Typical meshes generated by PolyMesher are shown in Figure 7.

Here, we again consider the numerical example presented in Section 7.1; namely, we let $\Omega=(0,1)^{2}$ and select $f=2 \pi^{2} \sin (\pi x) \sin (\pi y)$, so that $u=\sin (\pi x) \sin (\pi y)$. In Figure 8 we plot the error, measured in terms of both the $L^{2}(\Omega)$ norm and the DG-norm $|\|\cdot \mid\|$, against the square root of the number of degrees of freedom in the underlying finite element space $S_{\mathcal{T}}^{\mathbf{p}}$ for (uniform) $p$ between 1 and 4 . Here, we clearly observe that the quantities $\left\|u-u_{h}\right\|_{L^{2}(\Omega)}$ and $\left|\left\|u-u_{h} \mid\right\|\right.$ converge to zero at the optimal rates $\mathcal{O}\left(h^{p+1}\right)$ and $\mathcal{O}\left(h^{p}\right)$, respectively, as the mesh size $h$ tends to zero for each (fixed) $p$; these latter results clearly confirm the optimality of Theorem 5.2. In particular, we observe that the error in the underlying IP DGFEM is smaller when polygonal elements II are employed, when compared to the corresponding quantity computed based on exploiting either uniform square elements or the polygonal elements I; this behaviour is more pronounced when the error is computed with respect to the DG-norm. We remark that similar behaviour was observed in Ref. 48 when the DG-norm of the error was computed on irregular quadrilateral meshes constructed by randomly splitting each of the interior nodes by a displacement of up to $10 \%$ of the local mesh size. As in Ref. 48 , we attribute the improvement in the computed error, when polygonal elements II are employed, to the increase in interelement communication. Indeed, uniform square elements may only communicate with their four immediate neighbours, while polygonal elements possess a much greater stencil due to the increase in the number of local element faces. We note that there is soem degradation of the computed error, when the sequence of irregular polygonal elements I are employed, as we would expect. 
December hp-DG' polygonal'elements`v13
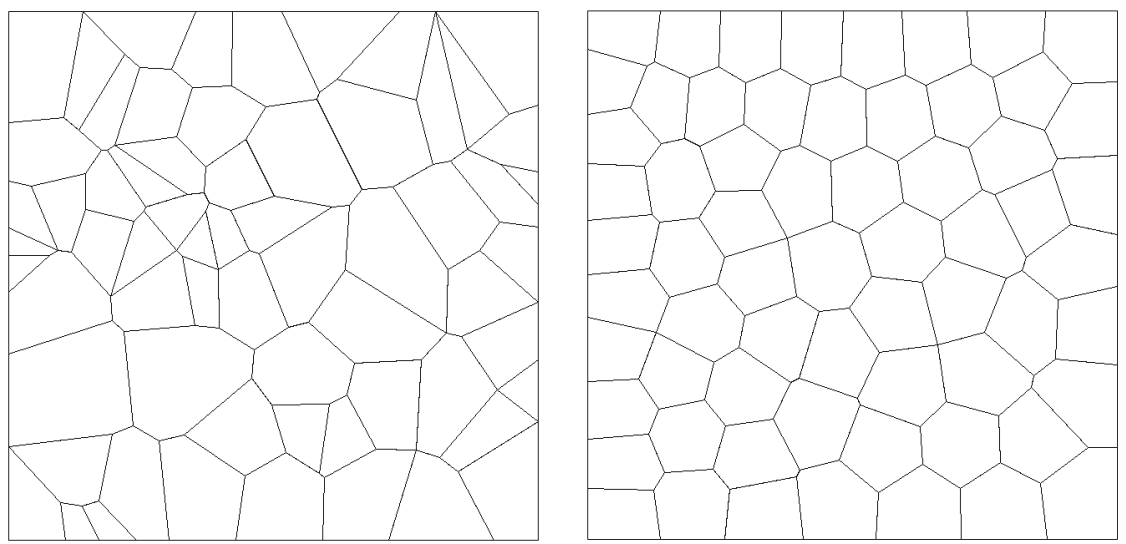

(a)
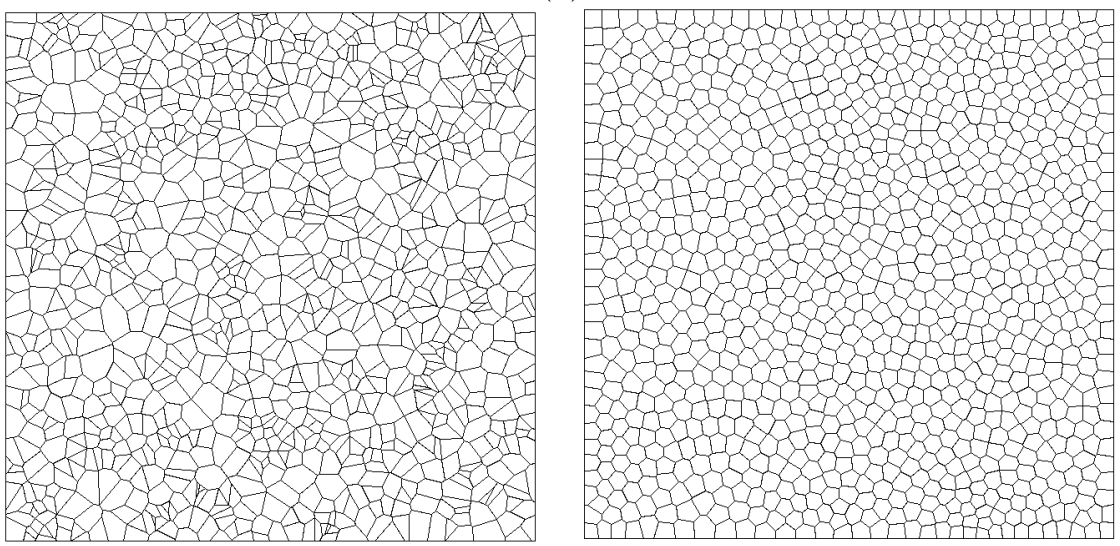

(b)

Fig. 7. Example 3. Polygonal element meshes generated using PolyMesher. Left: No mesh smoothing (Polygonal Elements I); Right: With 20 smoothing steps (Polygonal Elements II); (a) Mesh with 64 elements; (b) Mesh with 1024 elements.

Finally, we investigate the convergence of the IP DGFEM under $p$-refinement for fixed $h$. To this end, in Figure 9 we plot the DG-norm of the error against $p$ on five different square and polygonal meshes. In each case, we observe that on the linear-log scale, the convergence plots become straight lines as the degree of the approximating polynomial is increased, thereby indicating exponential convergence in $p$.

\section{Concluding Remarks}

We have studied the $h p$-version of the IP DGFEM for second-order elliptic partial differential equations, based on employing general computational meshes consisting of polygonal/polyhedral elements. Within this scheme polynomial bases are ex- 
December 2

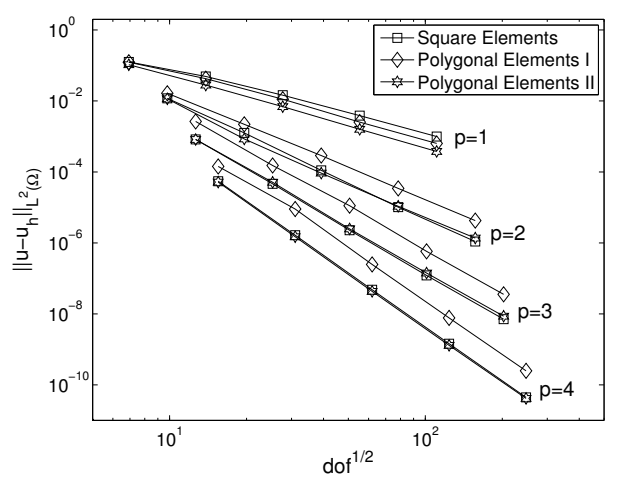

(a)

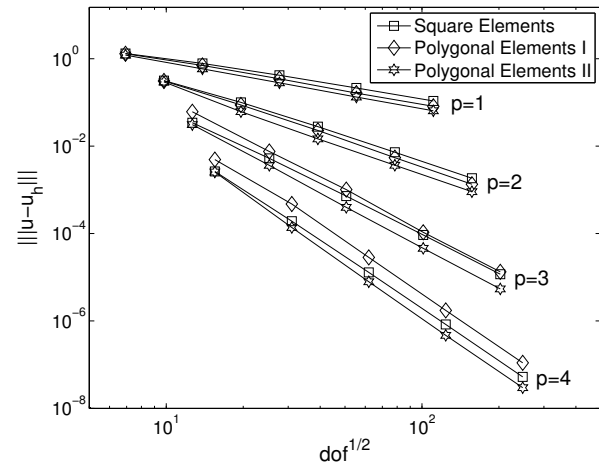

(b)

Fig. 8. Example 3. Convergence of the IP DGFEM with $h$-refinement: (a) $\left\|u-u_{h}\right\|_{L^{2}(\Omega)}$; (b) $\left\|u-u_{h} \mid\right\|$.

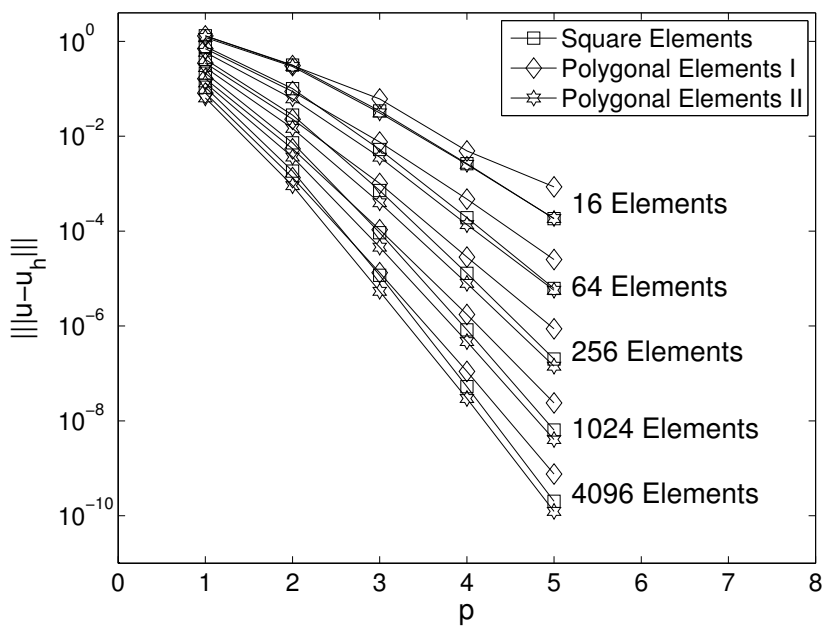

Fig. 9. Example 3. Convergence of the IP DGFEM with $p$-refinement.

ploited within the physical coordinate space, without the need to map from a given reference or canonical frame. This approach is advantageous from the point of view that only element spaces consisting of polynomials of total degree $p$ are sufficient to guarantee optimal convergence of the underlying method. On tensor-product meshes, the resulting scheme has been shown to, not only be computationally more efficient than standard DGFEMs based on employing full (mapped) tensor-product polynomial spaces, but also provide a competitive alternative to CGFEM, under $p$-enrichment. 


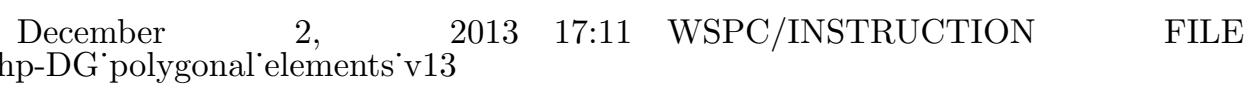

hp-Version discontinuous Galerkin methods on polygonal and polyhedral meshes

The a priori error analysis presented in this article extends and generalizes the error bounds derived in Ref. 8 for composite DGFEMs in a number of ways. In particular, the careful use of $h p$-version inverse inequalities and $h p$-version approximation properties, leads to an a priori error bound whose validity does not require any angle conditions, or any conditions on the relative sizes of the elemental interfaces on the polygonal/polyhedral mesh. Instead, the polygonal/polyhedral mesh is required to satisfy two weak assumptions (cf., Assumptions 4.1 and 4.2 which asserts in particular the existence of a shape-regular simplicial covering) for the error analysis to be valid. The resulting $h p$-version a priori bound is formally in accordance with respect to standard error estimates (for meshes consisting of standard simplicial/tensor-product elements) in the literature.

\section{References}

1. P.R. Amestoy, I.S. Duff, J. Koster, and J.-Y. L'Excellent. A fully asynchronous multifrontal solver using distributed dynamic scheduling. SIAM J. Matrix Anal. Appl., 23(1):15-41, 2001.

2. P.R. Amestoy, I.S. Duff, and J.-Y. L'Excellent. Multifrontal parallel distributed symmetricand unsymmetric solvers. Comput. Methods Appl. Mech. Eng., 184:501-520, 2000 .

3. P.R. Amestoy, A. Guermouche, J.-Y. L'Excellent, and S. Pralet. Hybrid scheduling for the parallel solution of linear systems. Parallel Computing, 32(2):136-156, 2006.

4. P.F. Antonietti and B. Ayuso. Schwarz domain decomposition preconditioners for discontinuous Galerkin approximations of elliptic problems: non-overlapping case. M2AN Math. Model. Numer. Anal., 41(1):21-54, 2007.

5. P.F. Antonietti and B. Ayuso. Multiplicative Schwarz methods for discontinuous Galerkin approximations of elliptic problems. M2AN Math. Model. Numer. Anal., 42(3):443-469, 2008.

6. P.F. Antonietti and B. Ayuso. Two-level schwarz preconditioners for super penalty discontinuous Galerkin methods. Commun. Comput. Phys., 5(2-4):398-412, 2009.

7. P.F. Antonietti, S. Giani, and P. Houston. Domain decomposition preconditioners for discontinuous Galerkin methods for elliptic problems on complicated domains. J. Sci. Comput., 2013. Online.

8. P.F. Antonietti, S. Giani, and P. Houston. $h p$-Version composite discontinuous Galerkin methods for elliptic problems on complicated domains. SIAM J. Sci. Comput., 35(3):A1417-A1439, 2013.

9. P.F. Antonietti and P. Houston. A class of domain decomposition preconditioners for $h p$-discontinuous Galerkin finite element methods. J. Sci. Comp., 46(1):124-149, 2011.

10. P.F. Antonietti and P. Houston. Preconditioning high-order discontinuous Galerkin discretizations of elliptic problems. In R. Bank, M. Holst, O. Widlund, and J. Xu, editors, Domain Decomposition Methods in Science and Engineering XX. Lecture Notes in Computational Science and Engineering, Vol. 91. Springer-Verlag, 2013.

11. D.N. Arnold. An interior penalty finite element method with discontinuous elements. SIAM J. Numer. Anal., 19(4):742-760, 1982.

12. D.N. Arnold, D. Boffi, and R.S. Falk. Approximation by quadrilateral finite elements. Math. Comp., 71(239):909-922, March 2002.

13. D.N. Arnold, D. Boffi, R.S. Falk, and L. Gastaldi. Finite element approximation on quadrilateral meshes. Commun. Numer. Meth. Engrg., 17(11):805-812, 2001. 


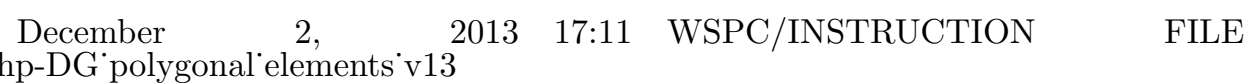

14. D.N. Arnold, F. Brezzi, B. Cockburn, and L.D. Marini. Unified analysis of discontinuous Galerkin methods for elliptic problems. SIAM J. Numer. Anal., 39:1749-1779, 2001.

15. J.P. Aubin. Approximation des problèmes aux limites non homogènes pour des opérateurs non linéaires. J. Math. Anal. Appl., 30:510-521, 1970.

16. I. Babuška and J. E. Osborn. Generalized finite element methods: their performance and their relation to mixed methods. SIAM J. Numer. Anal., 20(3):510-536, 1983.

17. I. Babuška and M. Suri. The $h-p$ version of the finite element method with quasiuniform meshes. RAIRO Modél. Math. Anal. Numér., 21(2):199-238, 1987.

18. I. Babuška and M. Suri. The optimal convergence rate of the $p$-version of the finite element method. SIAM J. Numer. Anal., 24(4):750-776, 1987.

19. I. Babuška. The finite element method with penalty. Math. Comp., 27(122):221-228, 1973.

20. G.A. Baker. Finite element methods for elliptic equations using nonconforming elements. Math. Comp., 31(137):45-59, 1977.

21. F. Bassi, L. Botti, and A. Colombo. An attempt to be mesh free, agglomeration based physical frame DG discretizations. Math. Models Methods Appl. Sci., Submitted for publication.

22. F. Bassi, L. Botti, A. Colombo, D.A. Di Pietro, and P. Tesini. On the flexibility of agglomeration based physical space discontinuous Galerkin discretizations. J. Comput. Phys., 231(1):45-65, 2012.

23. F. Bassi, L. Botti, A. Colombo, and S. Rebay. Agglomeration based discontinuous Galerkin discretization of the Euler and Navier-Stokes equations. Comput. \& Fluids, 61:77-85, 2012.

24. L. Beirão da Veiga, F. Brezzi, A. Cangiani, G. Manzini, L.D. Marini, and A. Russo. Basic principles of virtual element methods. Math. Models Methods Appl. Sci., 23(1):199$214,2013$.

25. L. Beirão da Veiga, J. Droniou, and G. Manzini. A unified approach for handling convection terms in finite volumes and mimetic discretization methods for elliptic problems. IMA J. Numer. Anal., 31(4):1357-1401, 2011.

26. L. Beirão da Veiga, K. Lipnikov, and G. Manzini. Arbitrary-order nodal mimetic discretizations of elliptic problems on polygonal meshes. SIAM J. Numer. Anal., 49(5):1737-1760, 2011.

27. L. Beirão da Veiga and G. Manzini. A virtual element method with arbitrary regularity. IMA J. Numer. Anal., in press, 2013.

28. F. Brezzi, A. Buffa, and K. Lipnikov. Mimetic finite differences for elliptic problems. M2AN Math. Model. Numer. Anal., 43(2):277-295, 2009.

29. F. Brezzi, K. Lipnikov, and M. Shashkov. Convergence of the mimetic finite difference method for diffusion problems on polyhedral meshes. SIAM J. Numer. Anal., 43(5):1872-1896 (electronic), 2005.

30. F. Brezzi, K. Lipnikov, and V. Simoncini. A family of mimetic finite difference methods on polygonal and polyhedral meshes. Math. Models Methods Appl. Sci., 15(10):15331551, 2005.

31. E. Burman and P. Hansbo. Fictitious domain finite element methods using cut elements: I. A stabilized Lagrange multiplier method. Comput. Methods Appl. Mech. Engrg., 199:2680-2686, 2010.

32. E. Burman and P. Hansbo. An interior-penalty-stabilized Lagrange multiplier method for the finite-element solution of elliptic interface problems. IMA J. Numer. Anal., 30:870-885, 2010.

33. E. Burman and P. Hansbo. Fictitious domain finite element methods using cut ele- 


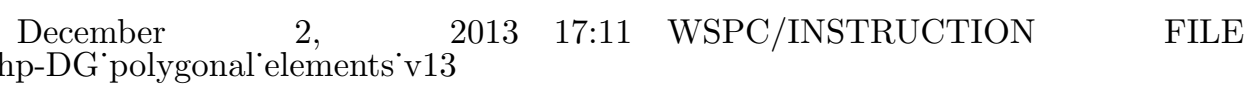

hp-Version discontinuous Galerkin methods on polygonal and polyhedral meshes

ments: II. A stabilized Nitsche method. Appl. Numer. Math., 62:328-341, 2012.

34. A. Cangiani, G. Manzini, and A. Russo. Convergence analysis of the mimetic finite difference method for elliptic problems. SIAM J. Numer. Anal., 47(4):2612-2637, 2009.

35. A. Chernov. Optimal convergence estimates for the trace of the polynomial $L^{2}$ projection operator on a simplex. Math. Comp., 81(278):765-787, 2012.

36. P.G. Ciarlet. The finite element method for elliptic problems. North-Holland Publishing Co., Amsterdam, 1978. Studies in Mathematics and its Applications, Vol. 4.

37. B. Cockburn. An introduction to the discontinuous Galerkin method for convectiondominated problems. In Advanced numerical approximation of nonlinear hyperbolic equations (Cetraro, 1997), pages 151-268. Springer, Berlin, 1998.

38. B. Cockburn, G.E. Karniadakis, and C.-W. Shu, editors. Discontinuous Galerkin methods. Springer-Verlag, Berlin, 2000. Theory, computation and applications, Papers from the 1st International Symposium held in Newport, RI, May 24-26, 1999.

39. D.A. Di Pietro and A. Ern. Mathematical aspects of discontinuous Galerkin methods, volume 69 of Mathématiques \& Applications (Berlin) [Mathematics $\&$ Applications]. Springer, Heidelberg, 2012.

40. T.-P. Fries and T. Belytschko. The extended/generalized finite element method: an overview of the method and its applications. Internat. J. Numer. Methods Engrg., 84(3):253-304, 2010.

41. E.H. Georgoulis. Inverse-type estimates on $h p$-finite element spaces and applications. Math. Comp., 77(261):201-219 (electronic), 2008.

42. S. Giani and P. Houston. Domain decomposition preconditioners for discontinuous Galerkin discretizations of compressible fluid flows. Submitted for publication.

43. S. Giani and P. Houston. $h p$-Adaptive composite discontinuous Galerkin methods for elliptic problems on complicated domains. Submitted for publication.

44. W. Hackbusch and S.A. Sauter. Composite finite elements for problems containing small geometric details. Part II: Implementation and numerical results. Comput. Visual Sci., 1:15-25, 1997.

45. W. Hackbusch and S.A. Sauter. Composite finite elements for the approximation of PDEs on domains with complicated micro-structures. Numer. Math., 75:447-472, 1997.

46. J.S. Hesthaven and T. Warburton. Nodal discontinuous Galerkin methods, volume 54 of Texts in Applied Mathematics. Springer, New York, 2008. Algorithms, analysis, and applications.

47. P. Houston, D. Schötzau, and T.P. Wihler. Energy norm a posteriori error estimation of $h p$-adaptive discontinuous Galerkin methods for elliptic problems. Math. Models Methods Appl. Sci., 17(1):33-62, 2007.

48. P. Houston, C. Schwab, and E. Süli. Stabilized $h p$-finite element methods for first-order hyperbolic problems. SIAM J. Numer. Anal., 37(5):1618-1643 (electronic), 2000.

49. P. Houston, C. Schwab, and E. Süli. Discontinuous $h p$-finite element methods for advection-diffusion-reaction problems. SIAM J. Numer. Anal., 39(6):2133-2163 (electronic), 2002.

50. P. Houston and E. Süli. A note on the design of $h p$-adaptive finite element methods for elliptic partial differential equations. Comput. Methods Appl. Mech. Engrg., 194(25):229-243, 2005 .

51. A. Johansson and M.G. Larson. A high order discontinuous Galerkin Nitsche method for elliptic problems with fictitious boundary. Numer. Math., 123(4):607-628, 2013.

52. P. Lesaint and P.-A. Raviart. On a finite element method for solving the neutron transport equation. In Mathematical aspects of finite elements in partial differential equations (Proc. Sympos., Math. Res. Center, Univ. Wisconsin, Madison, Wis., 1974), 


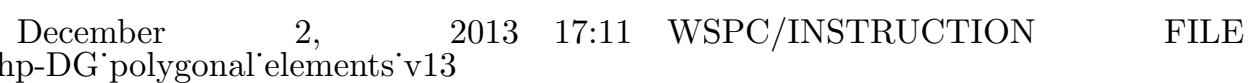

pages 89-123. Publication No. 33. Math. Res. Center, Univ. of Wisconsin-Madison, Academic Press, New York, 1974.

53. J.-L. Lions. Problèmes aux limites non homogènes à donées irrégulières: Une méthode d'approximation. In Numerical Analysis of Partial Differential Equations (C.I.M.E. 2 Ciclo, Ispra, 1967), Edizioni Cremonese, Rome, pages 283-292. 1968.

54. K. Lipnikov, D. Vassilev, and I. Yotov. Discontinuous Galerkin and mimetic finite difference methods for coupled Stokes-Darcy flows on polygonal and polyhedral grids. Numer. Math., pages 1-40, 2013.

55. A. Massing. Analysis and implementation of Finite Element Methods on overlapping and Fictitious Domains. PhD thesis, University of Oslo, 2012.

56. R. Muñoz-Sola. Polynomial liftings on a tetrahedron and applications to the $h-p$ version of the finite element method in three dimensions. SIAM J. Numer. Anal., 34(1):282-314, 1997.

57. S. Natarajan, S. Bordas, and D.R. Mahapatra. Numerical integration over arbitrary polygonal domains based on Schwarz-Christoffel conformal mapping. Internat. J. Numer. Methods Engrg., 80(1):103-134, 2009.

58. J. Nitsche. Über ein Variationsprinzip zur Lösung von Dirichlet Problemen bei Verwendung von Teilräumen, die keinen Randbedingungen unterworfen sind. Abh. Math. Sem. Uni. Hamburg, 36:9-15, 1971.

59. I. Perugia and D. Schötzau. An $h p$-analysis of the local discontinuous Galerkin method for diffusion problems. J. Sci. Comput., 17(1-4):561-571, 2002.

60. W.H. Reed and T.R. Hill. Triangular mesh methods for the neutron transport equation. Technical Report LA-UR-73-479 Los Alamos Scientific Laboratory, 1973.

61. B. Rivière. Discontinuous Galerkin methods for solving elliptic and parabolic equations, volume 35 of Frontiers in Applied Mathematics. Society for Industrial and Applied Mathematics (SIAM), Philadelphia, PA, 2008. Theory and implementation.

62. B. Rivière, M.F. Wheeler, and V. Girault. Improved energy estimates for interior penalty, constrained and discontinuous Galerkin methods for elliptic problems. I. Comput. Geosci., 3(3-4):337-360 (2000), 1999.

63. C. Schwab. $p$ - and hp- finite element methods: Theory and applications in solid and fluid mechanics. Oxford University Press: Numerical mathematics and scientific computation, 1998.

64. P. Solin, K. Segeth, and I. Dolezel. Higher-order finite element methods. Studies in advanced mathematics. Chapman \& Hall/CRC, Boca Raton, London, 2004.

65. E.M. Stein. Singular Integrals and Differentiability Properties of Functions. Princeton, University Press, Princeton, N.J., 1970.

66. G. Strang and G.J. Fix. An analysis of the finite element method. Prentice-Hall Inc., Englewood Cliffs, N. J., 1973. Prentice-Hall Series in Automatic Computation.

67. N. Sukumar and A. Tabarraei. Conforming polygonal finite elements. Internat. J. Numer. Methods Engrg., 61(12):2045-2066, 2004.

68. A. Tabarraei and N. Sukumar. Extended finite element method on polygonal and quadtree meshes. Comput. Methods Appl. Mech. Engrg., 197(5):425-438, 2007.

69. C. Talischi, G.H. Paulino, A. Pereira, and I.F.M. Menezes. Polymesher: a generalpurpose mesh generator for polygonal elements written in Matlab. Struct. Multidisc. Optim., 45:309328, 2012.

70. M.F. Wheeler. An elliptic collocation-finite element method with interior penalties. SIAM J. Numer. Anal., 15(1):152-161, 1978.

71. T.P. Wihler, P. Frauenfelder, and C. Schwab. Exponential convergence of the $h p$ DGFEM for diffusion problems. Comput. Math. Appl., 46:183-205, 2003.

72. D. Wirasaet, E.J. Kubatko, C.E. Michoski, S. Tanaka, J.J. Westerink, and C. Daw- 
December $\quad 2, \quad 2013 \quad 17: 11 \quad$ WSPC/INSTRUCTION $\quad$ FILE hp-DG polygonal'elements'v13

hp-Version discontinuous Galerkin methods on polygonal and polyhedral meshes 33

son. Discontinuous galerkin methods with nodal and hybrid modal/nodal triangular, quadrilateral, and polygonal elements for nonlinear shallow water flow. Comput. Methods Appl. Mech. Engrg., In review. 